\title{
Bi-unitary multiperfect numbers, IV(b)
}

\author{
Pentti Haukkanen ${ }^{1}$ and Varanasi Sitaramaiah $^{2, *}$ \\ ${ }^{1}$ Faculty of Information Technology and Communication Sciences, \\ FI-33014 Tampere University, Finland \\ e-mail: pentti.haukkanen@tuni.fi \\ 2 1/194e, Poola Subbaiah Street, Taluk Office Road, Markapur \\ Prakasam District, Andhra Pradesh, 523316 India
}

Dedicated to the memory of Prof. D. Suryanarayana

Received: 17 July $2020 \quad$ Revised: 3 November $2020 \quad$ Accepted: 6 November 2020

\begin{abstract}
A divisor $d$ of a positive integer $n$ is called a unitary divisor if $\operatorname{gcd}(d, n / d)=1$; and $d$ is called a bi-unitary divisor of $n$ if the greatest common unitary divisor of $d$ and $n / d$ is unity. The concept of a bi-unitary divisor is due to D. Surynarayana (1972). Let $\sigma^{* *}(n)$ denote the sum of the bi-unitary divisors of $n$. A positive integer $n$ is called a bi-unitary multiperfect number if $\sigma^{* *}(n)=k n$ for some $k \geq 3$. For $k=3$ we obtain the bi-unitary triperfect numbers.

Peter Hagis (1987) proved that there are no odd bi-unitary multiperfect numbers. The present paper is part IV(b) in a series of papers on even bi-unitary multiperfect numbers. In parts I, II and III we considered bi-unitary triperfect numbers of the form $n=2^{a} u$, where $1 \leq a \leq 6$ and $u$ is odd. In part IV(a) we solved partly the case $a=7$. We proved that if $n$ is a bi-unitary triperfect number of the form $n=2^{7} \cdot 5^{b} .17^{c} . v$, where $(v, 2.5 .17)=1$, then $b \geq 2$. We then solved completely the case $b=2$. In the present paper we give some partial results concerning the case $b \geq 3$ under the assumption $3 \nmid n$.
\end{abstract}

Keywords: Perfect numbers, Triperfect numbers, Multiperfect numbers, Bi-unitary analogues. 2010 Mathematics Subject Classification: 11A25.

\section{Introduction}

Throughout this paper, all lower case letters denote positive integers; $p$ and $q$ denote primes. The letters $u, v$ and $w$ are reserved for odd numbers.

\footnotetext{
* Prof. Varanasi Sitaramaiah passed away on 2 October 2020.
} 
A divisor $d$ of $n$ is called a unitary divisor if $\operatorname{gcd}(d, n / d)=1$. If $d$ is a unitary divisor of $n$, we write $d \| n$. A divisor $d$ of $n$ is called a bi-unitary divisor if $(d, n / d)^{* *}=1$, where the symbol $(a, b)^{* *}$ denotes the greatest common unitary divisor of $a$ and $b$. The concept of a bi-unitary divisor is due to D. Suryanarayana (cf. [7]). Let $\sigma^{* *}(n)$ denote the sum of bi-unitary divisors of $n$. The function $\sigma^{* *}(n)$ is multiplicative, that is, $\sigma^{* *}(1)=1$ and $\sigma^{* *}(m n)=\sigma^{* *}(m) \sigma^{* *}(n)$ whenever $(m, n)=1$. If $p^{\alpha}$ is a prime power and $\alpha$ is odd, then every divisor of $p^{\alpha}$ is a bi-unitary divisor; if $\alpha$ is even, each divisor of $p^{\alpha}$ is a bi-unitary divisor except for $p^{\alpha / 2}$. Hence

$$
\sigma^{* *}\left(p^{\alpha}\right)=\left\{\begin{array}{l}
\sigma\left(p^{\alpha}\right)=\frac{p^{\alpha+1}-1}{p-1} \text { if } \alpha \text { is odd } \\
\sigma\left(p^{\alpha}\right)-p^{\alpha / 2} \text { if } \alpha \text { is even. }
\end{array}\right.
$$

If $\alpha$ is even, say $\alpha=2 k$, then $\sigma^{* *}\left(p^{\alpha}\right)$ can be simplified to

$$
\sigma^{* *}\left(p^{\alpha}\right)=\left(\frac{p^{k}-1}{p-1}\right) \cdot\left(p^{k+1}+1\right) .
$$

From (1.3), it is not difficult to observe that $\sigma^{* *}(n)$ is odd only when $n=1$ or $n=2^{\alpha}$.

The concept of a bi-unitary perfect number was introduced by C. R. Wall [8]; a positive integer $n$ is called a bi-unitary perfect number if $\sigma^{* *}(n)=2 n$. C. R. Wall [8] proved that there are only three bi-unitary perfect numbers, namely 6,60 and 90. A positive integer $n$ is called a bi-unitary multiperfect number if $\sigma^{* *}(n)=k n$ for some $k \geq 3$. For $k=3$ we obtain the bi-unitary triperfect numbers.

Peter Hagis [1] proved that there are no odd bi-unitary multiperfect numbers. Our present paper is part VI(b) in a series of papers on even bi-unitary multiperfect numbers. In parts I, II and III (see $[2,3,4]$ ) we considered bi-unitary triperfect numbers of the form $n=2^{a} u$, where $1 \leq a \leq 6$ and $u$ is odd. In part IV(a) we solved partly the case $a=7$. We proved that if $n$ is a bi-unitary triperfect number of the form $n=2^{7} \cdot 5^{b} \cdot 17^{c} . v$, where $(v, 2.5 .17)=1$, then $b \geq 2$. We then solved completely the case $b=2$. We proved that in this case $c$ has to equal 1 and further showed that $n=2^{7} \cdot 3^{2} \cdot 5^{2} \cdot 7 \cdot 13.17=44553600$ is the only bi-unitary triperfect number of the form considered there. In this paper we examine the case $b \geq 3$ with the restriction that $3 \nmid n$. We present some necessary conditions for triperfect numbers.

For a general account on various perfect-type numbers, we refer to [6].

\section{Preliminaries}

We assume that the reader has parts I, II, III, IV(a) (see [2, 3, 4, 5]) available. We, however, recall Lemmas 2.1 to 2.6 from part IV(a), because they are so important also here.

Lemma 2.1. (I) If $\alpha$ is odd, then for any prime $p$,

$$
\frac{\sigma^{* *}\left(p^{\alpha}\right)}{p^{\alpha}}>\frac{\sigma^{* *}\left(p^{\alpha+1}\right)}{p^{\alpha+1}}
$$

(II) For any $\alpha \geq 2 \ell-1$ and any prime $p$,

$$
\frac{\sigma^{* *}\left(p^{\alpha}\right)}{p^{\alpha}} \geq\left(\frac{1}{p-1}\right)\left(p-\frac{1}{p^{2 \ell}}\right)-\frac{1}{p^{\ell}}=\frac{1}{p^{2 \ell}}\left(\frac{p^{2 \ell+1}-1}{p-1}-p^{\ell}\right)
$$


(III) If $p$ is any prime and $\alpha$ is a positive integer, then

$$
\frac{\sigma^{* *}\left(p^{\alpha}\right)}{p^{\alpha}}<\frac{p}{p-1}
$$

Remark 2.1. (I) and (III) of Lemma 2.1 are mentioned in C. R. Wall [8]; (II) of Lemma 2.1 has been used by him [8] without explicitly stating it.

Lemma 2.2. Let $a>1$ be an integer not divisible by an odd prime $p$ and let $\alpha$ be a positive integer. Let $r$ denote the least positive integer such that $a^{r} \equiv 1\left(\bmod p^{\alpha}\right)$; then $r$ is usually denoted by $\operatorname{ord}_{p^{\alpha}}$ a. We have the following properties:

(i) If $r$ is even, then $s=r / 2$ is the least positive integer such that $a^{s} \equiv-1\left(\bmod p^{\alpha}\right)$. Also, $a^{t} \equiv-1\left(\bmod p^{\alpha}\right)$ for a positive integer $t$ if and only if $t=s u$, where $u$ is odd.

(ii) If $r$ is odd, then $p^{\alpha} \nmid a^{t}+1$ for any positive integer $t$.

Remark 2.2. Let $a, p, r$ and $s=r / 2$ be as in Lemma 2.2 ( $\alpha=1)$. Then $p \mid a^{t}-1$ if and only if $r \mid t$. If $t$ is odd and $r$ is even, then $r \nmid t$. Hence $p \nmid a^{t}-1$. Also, $p \mid a^{t}+1$ if and only if $t=s u$, where $u$ is odd. In particular if $t$ is even and $s$ is odd, then $p \nmid a^{t}+1$. In order to check the divisibility of $a^{t}-1$ (when $t$ is odd) by an odd prime $p$, we can confine to those $p$ for which $\operatorname{ord}_{p} a$ is odd. Similarly, for examining the divisibility of $a^{t}+1$ by $p$ when $t$ is even we need to consider primes $p$ with $s=\operatorname{ord}_{p} a / 2$ even.

Lemma 2.3. Let $k$ be odd and $k \geq 3$. Let $p \neq 5$.

(a) If $p \in[3,2520]-\{11,19,31,71,181,829,1741\}$, ord 5 is odd and $p \mid 5^{k}-1$, then we can find a prime $p^{\prime}$ (depending on $p$ ) such that $p^{\prime} \mid \frac{5^{k}-1}{4}$ and $p^{\prime} \geq 2521$.

(b) If $q \in[3,2520]-\{13,313,601\}, s=\frac{1}{2}$ ord $_{q} 5$ is even and $q \mid 5^{k+1}+1$, then we can find $a$ prime $q^{\prime}$ (depending on $q$ ) such that $q^{\prime} \mid \frac{5^{k+1}+1}{2}$ and $q^{\prime} \geq 2521$.

Lemma 2.4. Let $k$ be odd and $k \geq 3$. Let $p \neq 7$.

(a) If $p \in[3,2520]-\{3,19,37,1063\}, r=\operatorname{ord}_{p} 7$ is odd and $p \mid 7^{k}-1$, then we can find $a$ prime $p^{\prime}$ (depending on $p$ ) such that $p^{\prime} \mid \frac{7^{k}-1}{6}$ and $p^{\prime} \geq 2521$.

(b) If $q \in[3,1193]-\{5,13,181,193,409\}, s=\frac{1}{2} \operatorname{ord}_{q} 7$ is even and $q \mid 7^{k+1}+1$, then we can find a prime $q^{\prime}$ (depending on $q$ ) such that $q^{\prime} \mid \frac{7^{k+1}+1}{2}$ and $q^{\prime}>1193$.

Lemma 2.5. Let $k$ be odd and $k \geq 3$. Let $p \neq 13$.

(a) If $p \in[3,293]-\{3,61\}, r=\operatorname{ord}_{p} 13$ is odd and $p \mid 13^{k}-1$, then we can find a prime $p^{\prime}$ (depending on $p$ ) such that $p^{\prime} \mid \frac{13^{k}-1}{12}$ and $p^{\prime} \geq 293$.

(b) If $q \in[3,293]-\{5,17\}, s=\frac{1}{2} \operatorname{ord}_{q} 13$ is even and $q \mid 13^{k+1}+1$, then we can find a prime $q^{\prime}$ (depending on $q$ ) such that $q^{\prime} \mid \frac{13^{k+1}+1}{2}$ and $q^{\prime}>293$. 
Lemma 2.6. Let $k$ be odd and $k \geq 3$. Let $p \neq 17$.

(a) If $p \in[3,519]-\{307\}, r=\operatorname{ord}_{p} 17$ is odd and $p \mid 17^{k}-1$, then we can find a prime $p^{\prime}$ (depending on $p$ ) such that $p^{\prime} \mid \frac{17^{k}-1}{16}$ and $p^{\prime}>519$.

(b) If $q \in[3,519]-\{5,29\}, s=\frac{1}{2} \operatorname{ord}_{q} 17$ is even and $q \mid 17^{k+1}+1$, then we can find a prime $q^{\prime}$ (depending on $q$ ) such that $q^{\prime} \mid \frac{17^{k+1}+1}{2}$ and $q^{\prime}>519$.

Corollary 2.1. (I) If $k$ is odd and $k \geq 5$, then $\frac{17^{k}-1}{16}$ is divisible by an odd prime $p^{\prime}>519$.

(II) If $k$ is odd and $k \geq 3$, then $\frac{17^{k+1}+1}{2}$ is divisible by an odd prime $q^{\prime}>519$.

Proof. (I) Let $S_{17}=\left\{p \mid 17^{k}-1: p \in[3,519]-\{307\}\right.$ and $r=\operatorname{ord}_{p} 17$ is odd $\}$. By Lemma 2.6 (a), if $S_{17}$ is non-empty, the statement in (I) above holds.

Let $S_{17}$ be empty. Since $p \nmid 17^{k}-1$ if $\operatorname{ord}_{p} 17$ is even, it follows that $17^{k}-1$ is not divisible by any prime in $[3,519]$ except for possibly by 307 . As $32 \mid 17^{t}-1$ if and only if $t$ is even, $32 \nmid 17^{k}-1$ since $k$ is odd. Hence $16 \| 17^{k}-1$. Thus $\frac{17^{k}-1}{16}$ is odd; also it is $>1$, since $k \geq 5$.

If $307 \nmid 17^{k}-1$, then $\frac{17^{k}-1}{16}$ is divisible by none of the primes in $[3,519]$. If $p^{\prime} \mid \frac{17^{k}-1}{16}$, then $p^{\prime}>519$. This proves (I) in this case.

Assume that $307 \mid 17^{k}-1$. We claim that $17^{k}-1$ is divisible by an odd prime $\neq 307$. On the contrary, let $\frac{17^{k}-1}{16}=307^{\alpha}$. If $\alpha \geq 2$, then $307^{2} \mid 17^{k}-1$; this holds if and only if $921=3.307 \mid k$. In particular, 307| $k$. But 7036178437| $\frac{17^{307}-1}{16} \mid \frac{17^{k}-1}{16}=307^{\alpha}$, which is impossible. Hence $\alpha=1$ so that $\frac{17^{k}-1}{16}=307$ or $k=3$. But $k \geq 5$, by hypothesis. Hence we can find an odd prime $p^{\prime} \mid \frac{17^{k}-1}{16}$ and $p^{\prime} \neq 307$. Also, $p^{\prime}>519$.

The proof of (I) is complete.

(II) Consider the factor $17^{k+1}+1$, where $k$ is odd and $\geq 3$. Let

$$
T_{17}=\left\{q \mid 17^{k+1}+1: q \in[3,519]-\{5,29\} \text { and } s=\frac{1}{2} \operatorname{ord}_{p} 17 \text { is even }\right\} .
$$

By Lemma 2.6 (b), if $T_{17}$ is non-empty, then the statement in (II) holds. So we may assume that $T_{17}$ is empty. Since $q \nmid 17^{k+1}+1$ if $s=\frac{1}{2} \operatorname{ord}_{p} 17$ is not even, it follows that $17^{k+1}+1$ is not divisible by any prime in $[3,519]$ except for possibly 5 and 29 .

We may note that $5\left|17^{k+1}+1 \Longleftrightarrow k+1=2 u \Longleftrightarrow 29\right| 17^{k+1}+1$. Hence if $5 \nmid 17^{k+1}+1$, then $29 \nmid 17^{k+1}+1$ so that $\frac{17^{k+1}+1}{2}$ is not divisible by any prime in $[3,519]$. So if $q^{\prime} \mid \frac{17^{k+1}+1}{2}$, then $q^{\prime}>519$.

Suppose that $5 \mid 17^{k+1}+1$. Hence $29 \mid 17^{k+1}+1$. We now claim that $\frac{17^{k+1}+1}{2}$ is divisible by an odd prime $q^{\prime} \notin\{5,29\}$. On the contrary, let $\frac{17^{k+1}+1}{2}=5^{\alpha} \cdot 29^{\beta}$. If $\alpha \geq 2$, then $5^{2} \mid 17^{k+1}+1$; this holds if and only if $k+1=10 u$. Hence $17^{10}+1 \mid 17^{k+1}+1$.

Also, $17^{10}+1=2.5^{2} .29 .21881 .63541$. Thus, $21881\left|\frac{17^{10}+1}{2}\right| \frac{17^{k+1}+1}{2}=5^{\alpha} .29^{\beta}$, which is not possible. Hence $\alpha=1$.

Suppose that $\beta \geq 2$. Then $29^{2} \mid 17^{k+1}+1$; this is equivalent to $k+1=58 u$. Hence, $17^{58}+1 \mid 17^{k+1}+1$. Also,

$$
17^{58}+1=\{\{2,1\},\{5,1\},\{29,2\},\{4908077,1\},
$$

$\{5627688836691687811685586936872121257317104508544673081805033,1\}\}$. 
Thus $4908077\left|\frac{17^{58}+}{2}\right| \frac{17^{k+1}+1}{2}=5^{\alpha} .29^{\beta}$, which is impossible. Hence $\beta=1$.

We now have $\frac{17^{k+1}+1}{2}=5.29$ or $k=1$. But $k \geq 3$. This contradiction proves that we can find an odd prime $q^{\prime} \mid \frac{17^{k+1}+1}{2}$ and $q^{\prime} \notin\{5,29\}$. It follows that $q^{\prime} \notin[3,519]$. Hence $q^{\prime}>519$.

\section{Further results on bi-unitary triperfect numbers of the form $n=2^{7} u$}

Let $n$ be a bi-unitary triperfect number divisible unitarily by $2^{7}$ so that $\sigma^{* *}(n)=3 n$ and $n=2^{7} . u$, where $u$ is odd. Since $\sigma^{* *}\left(2^{7}\right)=2^{8}-1=255=3.5 .17$, using $n=2^{7} u$ in $\sigma^{* *}(n)=3 n$, we get the following equations:

$$
n=2^{7} \cdot 5^{b} \cdot 17^{c} \cdot v
$$

and

$$
2^{7} \cdot 5^{b-1} \cdot 17^{c-1} \cdot v=\sigma^{* *}\left(5^{b}\right) \cdot \sigma^{* *}\left(17^{c}\right) \cdot \sigma^{* *}(v),
$$

where $(v, 2.5 .17)=1$. Considering the parity of the function values of $\sigma^{* *}$ and applying multiplicativity of $\sigma^{* *}$, we conclude that $v$ has at most five odd prime factors.

In part IV(a) we proved that $b \geq 2$ in $(3.1 a)$ and solved completely the case $b=2$. These results were presented in Theorem 3.1.

Theorem 3.1. (a) If $n$ is as in (3.1a) and $n$ is a bi-unitary triperfect number, then $b \geq 2$. (b) If $b=2$, then $c=1$ and $n=44553600=2^{7} \cdot 3^{2} \cdot 5^{2} \cdot 7 \cdot 13.17$.

In part IV(a) we also presented the following remark.

Remark 3.1. Let $n$ be as given in (3.1a) and $b \geq 3$. Assume that $n$ is a bi-unitary triperfect number. Then $(3.1 b)$ is valid. Further suppose that $n$ is not divisible by 3 . If $b$ is odd or $4 \mid b$, then $3 \mid \sigma^{* *}\left(5^{b}\right)$. Also, if $c$ is odd or $4 \mid c$, then $9 \mid \sigma^{* *}\left(17^{c}\right)$. These are not possible in $(3.1 b)$, and therefore it follows that $b=2 k$ and $c=2 \ell$, where $k \geq 3$ and $\ell$ are odd. Hence $b \geq 6$ and $c \geq 2$.

In this paper we consider the case $b \geq 3$ with $3 \nmid n$ in more detail. In Theorems 3.2, 3.3 and 3.4 we present some necessary conditions for such triperfect numbers.

Theorem 3.2. Let $n$ be as given in (3.1a), where $b \geq 3$ and $3 \nmid n$. Then $n$ is not a bi-unitary triperfect number,

(a) if $n$ is divisible by $7^{3}$ and $17^{3}$;

(b) if $17^{2} \| n$ and $7^{3} \mid n$;

(c) if $17^{2} \| n$ and $d=1$ or $d=2$, where $7^{d} \| n$;

(d) if $17^{3} \mid n$ and $7 \| n$;

(e) if $17^{3}\left|n, 7^{2}\right| \mid n$ and $n$ is not divisible by 11 and 13 . 
Proof. We assume that $n$ is not divisible by $3,7^{d} \| n$ and $n$ is a bi-unitary triperfect number. From $(3.1 b)$, it follows that $v=7^{d} . w$, from $(3.1 a)$ and $(3.1 b)$, we have

$$
n=2^{7} \cdot 5^{b} \cdot 17^{c} \cdot 7^{d} \cdot w
$$

and

$$
2^{7} \cdot 5^{b-1} \cdot 17^{c-1} \cdot 7^{d} \cdot w=\sigma^{* *}\left(5^{b}\right) \cdot \sigma^{* *}\left(17^{c}\right) \cdot \sigma^{* *}\left(7^{d}\right) \cdot \sigma^{* *}(w),
$$

where

$$
(w, 2.3 .5 .7 .17)=1 \text { and } w \text { has not more than four odd prime factors. }
$$

Proof of $(a)$. By Remark 3.1, we can assume that $b \geq 6$. We have $\frac{\sigma^{* *}\left(5^{b}\right)}{5^{b}} \geq \frac{19406}{15625},(b \geq 5)$; $\frac{\sigma^{* *}\left(17^{c}\right)}{17^{c}} \geq \frac{88452}{83521},(c \geq 3)$, and $\frac{\sigma^{* *}\left(7^{d}\right)}{7^{d}} \geq \frac{2752}{2401},(d \geq 3)$. Hence for $c \geq 3$ and $d \geq 3$, by $(3.2 a)$, we have

$$
3=\frac{\sigma^{* *}(n)}{n} \geq \frac{255}{128} \cdot \frac{19406}{15625} \cdot \frac{88452}{83521} \cdot \frac{2752}{2401}=3.00348829>3,
$$

a contradiction. This proves (a).

Remark 3.2. In view of Theorem 3.2(a), we need to investigate the following cases. Case I: $c=2, d \geq 3$; Case II: $c=2, d=1$ or 2 ; Case III: $c \geq 3, d=1$ or 2 .

Proof of $(b)$. By hypothesis, $c=2$ and $d \geq 3$, we are dealing with Case I mentioned above. We have $\sigma^{* *}\left(17^{2}\right)=290=2.5 .29$. Taking $c=2$ in $(3.2 b)$, we see that $29 \mid w$. Let $w=29^{e} . w^{\prime}$. From $(3.2 a)$ and $(3.2 b)$, we have

$$
n=2^{7} \cdot 5^{b} \cdot 17^{2} \cdot 7^{d} \cdot 29^{e} \cdot w^{\prime},
$$

and

$$
2^{6} \cdot 5^{b-2} \cdot 17 \cdot 7^{d} \cdot 29^{e-1} \cdot w^{\prime}=\sigma^{* *}\left(5^{b}\right) \cdot \sigma^{* *}\left(7^{d}\right) \cdot \sigma^{* *}\left(29^{e}\right) \cdot \sigma^{* *}\left(w^{\prime}\right)
$$

where

$\left(w^{\prime}, 2\right.$ 3.5.7.17.29) $=1$ and $w^{\prime}$ has no more than three odd prime factors.

By Lemma 21.1, we have $\frac{\sigma^{* *}\left(5^{b}\right)}{5^{b}} \geq \frac{487656}{390625},(b \geq 7) ; \frac{\sigma^{* *}\left(7^{d}\right)}{7^{d}} \geq \frac{136914}{117649},(d \geq 5)$; and $\frac{\sigma^{* *}\left(29^{e}\right)}{29^{e}} \geq \frac{731700}{707281}(e \geq 3)$.

Hence when $b \geq 7, c=2, d \geq 5$ and $e \geq 3$, from (3.3a), we obtain

$$
3=\frac{\sigma^{* *}(n)}{n} \geq \frac{255}{128} \cdot \frac{487656}{390625} \cdot \frac{290}{289} \cdot \frac{136914}{117649} \cdot \frac{731700}{707281}=3.004585627>3,
$$

a contradiction. Hence when $b \geq 7, c=2, d \geq 5$, we have $e=1$ or $e=2$.

If $e=1$, from (3.3b) it follows that its left-hand side is divisible by 3 . This is false.

Let $e=2$. Since $\sigma^{* *}\left(29^{2}\right)=842=2.421$, taking $e=2$ in $(3.3 b)$, we see that $421 \mid w^{\prime}$. Let $w^{\prime}=421^{f} . w^{\prime \prime}$. From $(2.4 a)$ and $(2.4 b)$, we have

$$
n=2^{7} \cdot 5^{b} \cdot 17^{2} \cdot 7^{d} \cdot 29^{2} \cdot 421^{f} \cdot w^{\prime \prime}, \quad(b \geq 7, d \geq 5)
$$


and

$$
2^{5} \cdot 5^{b-2} \cdot 17 \cdot 7^{d} \cdot 29 \cdot 421^{f-1} \cdot w^{\prime \prime}=\sigma^{* *}\left(5^{b}\right) \cdot \sigma^{* *}\left(7^{d}\right) \cdot \sigma^{* *}\left(421^{f}\right) \cdot \sigma^{* *}\left(w^{\prime \prime}\right)
$$

where

$\left(w^{\prime \prime}, 2.3 .5 .7 .17 .29 .421\right)=1$ and $w^{\prime \prime}$ has not more than two odd prime factors.

We obtain a contradiction by examining the prime factors of $\sigma^{* *}\left(7^{d}\right)$.

(i) If $d$ is odd or $4 \mid d$, then $8 \mid \sigma^{* *}\left(7^{d}\right)$. From $(3.3 b)$, we at once have $w^{\prime \prime}=1$, so that from (3.3a), $n=2^{7} \cdot 5^{b} \cdot 17^{2} \cdot 7^{d} \cdot 29^{2} \cdot 421^{f}$ and so

$$
3=\frac{\sigma^{* *}(n)}{n}<\frac{255}{128} \cdot \frac{5}{4} \cdot \frac{290}{289} \cdot \frac{7}{6} \cdot \frac{842}{841} \cdot \frac{421}{420}=2.925742293<3,
$$

a contradiction.

(ii) Let $d=2 u$, where $u$ is odd and $\geq 3$ since $d \geq 5$. We have

$$
\sigma^{* *}\left(7^{d}\right)=\left(\frac{7^{u}-1}{6}\right) \cdot\left(7^{u+1}+1\right), \quad(u \geq 3 \text { and odd }) .
$$

We prove that

(A) $\frac{7^{u}-1}{6}$ is divisible by an odd prime $p^{\prime} \mid w^{\prime \prime}$ and $p^{\prime}>73$,

(B) $\frac{7^{u+1}+1}{2}$ is divisible by an odd prime $q^{\prime} \mid w^{\prime \prime}$ and $q^{\prime}>73$.

Proof of $(A)$. We apply Lemma 2.3(a) replacing the interval $[3,2520]$ by $[3,73]$. Let

$$
S_{7}^{\prime}=\left\{p \mid 7^{u}-1: p \in[3,73]-\{3,19,37\} \text { and } \operatorname{ord}_{p} 7 \text { is odd }\right\}
$$

By Lemma 2.3 (a), if $S_{7}^{\prime}$ is non-empty, then we can find an odd prime $p^{\prime} \mid \frac{7^{u}-1}{6}$ and $p^{\prime}>73$; and by $(3.4 b), p^{\prime} \mid w^{\prime \prime}$. In this case (A) follows quickly.

(iii) Suppose that $S_{7}^{\prime}$ is empty. Since $p \nmid 7^{u}-1$ if $\operatorname{ord}_{p} 7$ is even, it follows that $7^{u}-1$ is not divisible by any prime $p$ in $[3,73]$ except for possibly $p \in\{3,19,37\}$. We now discuss the divisibility of $7^{u}-1$ by $p \in\{3,19,37\}$.

(iv) Since $u$ is odd, 2|| $7^{u}-1$. Also, $3 \mid 7^{u}-1$ but $9 \nmid 7^{u}-1$. In fact, if $9 \mid 7^{u}-1$, then $3\left|\frac{7^{u}-1}{6}\right| \sigma^{* *}\left(7^{d}\right)$. It follows from $(3.4 b)$ that $3 \mid w^{\prime \prime}$. This is false. Hence $3 \| 7^{u}-1$.

(v) We have $19\left|7^{u}-1 \Longleftrightarrow 3\right| u \Longleftrightarrow 9 \mid 7^{u}-1$. By (iii) above since $9 \nmid 7^{u}-1$ it follows that $19 \nmid 7^{u}-1$.

(vi) We have $37\left|7^{u}-1 \Longleftrightarrow 9\right| u$. Also, $7^{9}-1=2.3^{3} \cdot 19.37 .1063$. Hence $37 \mid 7^{u}-1$ implies that $9\left|7^{9}-1\right| 7^{u}-1$. This cannot happen by (iii).

Thus from (iii)-(vi), $\frac{7^{u}-1}{6}$ is $>1$, odd and not divisible divisible by any prime in [3,73]. Hence if $p^{\prime} \mid \frac{7^{u}-1}{6}$, then $p^{\prime}>73$ and from (3.4b), $p^{\prime} \mid w^{\prime \prime}$. This proves (A).

Proof of $(B)$. We apply Lemma 2.3(b) replacing the interval $[3,2520]$ by $[3,73]$. Let

$$
T_{7}^{\prime}=\left\{q \mid 7^{u+1}+1: q \in[3,73]-\{3,5,13\} \text { and } s=\frac{1}{2} \operatorname{ord}_{q} 7 \text { is even }\right\} .
$$

If $T_{7}^{\prime}$ is non-empty, (B) follows immediately (cf. Lemma 2.3(b)). 
(vii) Assume that $T_{7}^{\prime}$ is empty. Since $q \nmid 7^{u+1}+1$ if $s=\frac{1}{2} \operatorname{ord}_{q} 7$ is not even, it follows that $\frac{7^{u+1}+1}{2}$ is divisible by any prime $q$ in $[3,73]$ except for possibly $q=3,5,13$.

(viii) Clearly, $2 \| 7^{u+1}+1$ and $3 \nmid 7^{u+1}+1$.

(ix) We have $13\left|7^{u+1}+1 \Longleftrightarrow u+1=6 v \Longleftrightarrow 181\right| 7^{u+1}+1, v$ being odd. Assume that $13 \mid 7^{u+1}+1$. Hence 181| $7^{u+1}+1$. From (3.4b), $w^{\prime \prime}$ is divisible by 13 and 181. By (A), $p^{\prime} \mid w^{\prime \prime}$. Thus $w^{\prime \prime}$ in $(3.4 b)$ is divisible by three distinct odd primes $p^{\prime}, 13$ and 181. This violates $(3.4 c)$. Hence $13 \nmid 7^{u+1}+1$.

(x) It remains to discuss the divisibility of $7^{u+1}+1$ by 5 . If $5 \nmid 7^{u+1}+1$, then from (vii)-(ix) it follows that $\frac{7^{u+1}+1}{2}$ is not divisible by any prime in $[3,73]$. If $q^{\prime} \mid \frac{7^{u+1}+1}{2}$, then $q^{\prime}>73$ and by $(3.4 b), q^{\prime} \mid w^{\prime \prime}$. This proves (B) in the case $5 \nmid 7^{u+1}+1$.

We may assume that $5 \mid 7^{u+1}+1$. This is equivalent to $u+1=2 v$. Hence $7^{2}+1 \mid 7^{u+1}+1$ and so $5^{2} \mid 7^{u+1}+1$. We now prove that $\frac{7^{u+1}+1}{2}$ is not divisible by 5 alone. Let $\frac{7^{u+1}+1}{2}=5^{\alpha}$, where $\alpha \geq 2$. If $\alpha \geq 3$, then $5^{3} \mid 7^{u+1}+1$. This is possible if and only if $u+1=10 v$. Hence $7^{10}+1 \mid 7^{u+1}+1$. Also, $7^{10}+1=2.5^{3} .281 .4021$. It follows that $281\left|\frac{7^{10}+1}{2}\right| \frac{7^{u+1}+1}{2}=5^{\alpha}$ and this cannot happen. Hence $\alpha=2$. Thus $\frac{7^{u+1}+1}{2}=5^{2}$ or $u=1$. But $u \geq 3$. Hence $\frac{7^{u+1}+1}{2}$ will be divisible by an odd prime say $q^{\prime} \neq 5$. From (vii)-(x), it follows that $q^{\prime} \notin[3,73]$. It is clear that $q^{\prime} \mid w^{\prime \prime}$. This proves (B) completely.

The odd primes $p^{\prime}$ and $q^{\prime}$ are distinct and hence we may assume that $p^{\prime} \geq 79$ and $q^{\prime} \geq 83$. From $(3.4 c), w^{\prime \prime}=\left(p^{\prime}\right)^{g} \cdot\left(q^{\prime}\right)^{h}$. From $(3.4 a)$, we have $n=2^{7} \cdot 5^{b} \cdot 17^{2} \cdot 7^{d} \cdot 29^{2} \cdot 421^{f} \cdot\left(p^{\prime}\right)^{g} \cdot\left(q^{\prime}\right)^{h}$. Hence

$$
3=\frac{\sigma^{* *}(n)}{n}<\frac{255}{128} \cdot \frac{5}{4} \cdot \frac{290}{289} \cdot \frac{7}{6} \cdot \frac{842}{841} \cdot \frac{421}{420} \cdot \frac{79}{78} \cdot \frac{83}{82}=2.999389027<3,
$$

a contradiction.

Thus when $c=2$, we cannot have $b \geq 7$ and $d \geq 5$. Hence we have either $\{c=2, b=6, d \geq 5\}$ or $\{c=2, b \geq 7, d=3$ or 4$\}$ or $\{c=2, b=6, d=3$ or 4$\}$, since already $b \geq 6$ and $d \geq 3$.

Let $\{c=2, b=6, d \geq 5\}$. We have $\sigma^{* *}\left(5^{6}\right)=2.31 .313$. Taking $b=6$ in $(3.3 b)$, it follows that $w^{\prime}$ is divisible by 31 and 313 . Let $w^{\prime}=31^{f} .313^{g} . w^{\prime \prime}$. Hence from $(3.3 a)$ and $(3.3 b)$ we obtain (after simplification),

$$
n=2^{7} \cdot 5^{6} \cdot 17^{2} \cdot 7^{d} \cdot 29^{e} \cdot 31^{f} \cdot 313^{g} \cdot w^{\prime \prime}, \quad(d \geq 5)
$$

and

$$
2^{5} \cdot 5^{4} \cdot 17 \cdot 7^{d} \cdot 29^{e-1} \cdot 31^{f-1} \cdot 313^{g-1} \cdot w^{\prime \prime}=\sigma^{* *}\left(7^{d}\right) \cdot \sigma^{* *}\left(29^{e}\right) \cdot \sigma^{* *}\left(31^{f}\right) \cdot \sigma^{* *}\left(313^{g}\right) \sigma^{* *}\left(w^{\prime \prime}\right),
$$

where

$$
\left(w^{\prime \prime}, 2 \cdot 3 \cdot 5 \cdot 7.17 .29 .31 .313\right)=1 \text { and } w^{\prime \prime} \text { has not more than one odd prime factors } .
$$

We have $\frac{\sigma^{* *}\left(5^{6}\right)}{5^{6}}=\frac{19406}{15625}$; by Lemma 2.1, $\frac{\sigma^{* *}\left(7^{d}\right)}{7^{d}} \geq \frac{136914}{117649} \quad(d \geq 5) ; \frac{\sigma^{* *}\left(29^{e}\right)}{29^{e}} \geq \frac{731700}{707281} \quad(e \geq$ $3)$ and $\frac{\sigma^{* *}\left(31^{f}\right)}{31^{f}} \geq \frac{953344}{923521} \quad(f \geq 3)$. 
From $(3.5 a)$, for $e \geq 3$ and $f \geq 3$, we have

$$
3=\frac{\sigma^{* *}(n)}{n} \geq \frac{255}{128} \cdot \frac{19406}{15625} \cdot \frac{290}{289} \cdot \frac{136914}{117649} \cdot \frac{731700}{707281} \cdot \frac{953344}{923521}=3.08567307873>3,
$$

a contradiction.

Hence $(e=1$ or 2$)$ or $(f=1$ or 2$)$.

Let $f=1$. We have $\sigma^{* *}(31)=32=2^{5}$. Hence taking $f=1$ in $(3.5 b)$, we see that its right-hand side is divisible by $2^{8}$ whereas $2^{5}$ is a unitary divisor of its left-hand side.

Let $f=2$. We have $\sigma^{* *}\left(31^{2}\right)=962=2.13 .37$. Taking $f=2$ in $(3.5 b)$, we find that $w^{\prime \prime}$ is divisible by 13 and 37 . This contradicts $(3.5 c)$.

Let $e=1$. We have $\sigma^{* *}(29)=30$. Taking $e=1$ in $(3.5 b)$, we see that its left-hand side is divisible by 3 . This cannot happen as $3 \nmid n$ by our assumption.

Let $e=2$. We have $\sigma^{* *}\left(29^{2}\right)=842=2.421$. Taking $e=2$ in $(3.5 b)$, we find that $421 \mid w^{\prime \prime}$. Let $w^{\prime \prime}=421^{h}$. From $(3.5 a)$ and $(3.5 b)$, we obtain

$$
n=2^{7} \cdot 5^{6} \cdot 17^{2} \cdot 7^{d} \cdot 29^{2} \cdot 31^{f} \cdot 313^{g} \cdot 421^{h}, \quad(d \geq 5)
$$

and

$$
2^{4} \cdot 5^{4} \cdot 17 \cdot 7^{d} \cdot 29 \cdot 31^{f-1} \cdot 313^{g-1} \cdot 421^{h-1}=\sigma^{* *}\left(7^{d}\right) \cdot \sigma^{* *}\left(31^{f}\right) \cdot \sigma^{* *}\left(313^{g}\right) \sigma^{* *}\left(421^{h}\right) .
$$

We obtain a contradiction by examining $\sigma^{* *}\left(7^{d}\right)$ as follows.

If $d$ is odd or $4 \mid d$, then $8 \mid \sigma^{* *}\left(7^{d}\right)$. This is not possible as in such a case $2^{6}$ is a factor of the right-hand side of $(3.6 b)$, while $2^{4}$ is a unitary divisor of its left-hand side.

Let $d=2 u$, where $u$ is odd. Then $u \geq 3$, since $d \geq 5$. We have

$$
\sigma^{* *}\left(7^{d}\right)=\left(\frac{7^{u}-1}{6}\right) \cdot\left(7^{u+1}+1\right) .
$$

(xi) Note that $\frac{7^{u}-1}{6}>1$ and odd. Also, it is not divisible by $5,17,313$ and 421 , since $u$ is odd (see Appendix $\mathrm{C}$ in [2]). It is not divisible by 7 trivially.

(xii) Assume that $29 \mid 7^{u}-1$. This is equivalent to $7 \mid u$. Hence $7^{7}-1 \mid 7^{u}-1$ so that

$$
4733\left|\frac{7^{7}-1}{6}\right| \frac{7^{u}-1}{6} \mid \sigma^{* *}\left(7^{d}\right) \text {. }
$$

This is not possible from $(3.6 b)$. Hence $29 \nmid 7^{u}-1$.

(xiii) We have $31\left|7^{u}-1 \Longleftrightarrow 15\right| u$. Hence $31 \mid 7^{u}-1$ implies that $3\left|\frac{7^{15}-1}{6}\right| \frac{7^{u}-1}{6} \mid \sigma^{* *}\left(7^{d}\right)$. It follows that 3 is a factor of the left-hand side of $(3.5 b)$. This is false. Hence $31 \nmid 7^{u}-1$.

From (xi)-(xiii) it follows that $\frac{7^{u}-1}{6}>1$, is odd and is not divisible by $5,7,17,29,31,313$ and 421. But this cannot happen from $(3.5 b)$, since $\frac{7^{u}-1}{6} \mid \sigma^{* *}\left(7^{d}\right)$.

Thus $c=2, b=6, d \geq 5$ leads to a contradiction.

Let $c=2$ and $d=3$. We have $\sigma^{* *}\left(7^{3}\right)=2^{4} \cdot 5^{2}$. Taking $d=3$ in $(3.3 b)$, we infer that $w^{\prime}=1$. Hence from $(3.3 a)$ and $(3.3 b)$, we have 


$$
n=2^{7} \cdot 5^{b} \cdot 17^{2} \cdot 7^{3} \cdot 29^{e},
$$

and

$$
2^{2} \cdot 5^{b-4} \cdot 17^{2} \cdot 7^{3} \cdot 29^{e}=\sigma^{* *}\left(5^{b}\right) \cdot \sigma^{* *}\left(29^{e}\right) .
$$

We obtain a contradiction by examining $\sigma^{* *}\left(5^{b}\right)$. By Remark 3.1, we can take $b=2 k$ where $k$ is odd and $\geq 3$. We have

$$
\sigma^{* *}\left(5^{b}\right)=\left(\frac{5^{k}-1}{4}\right) \cdot\left(5^{k+1}+1\right) .
$$

The factor $\frac{5^{k}-1}{4}>1$, is odd and is not divisible by 7,17 and 29 , since $k$ is odd and $\geq 3$; it is not divisible by 5 , trivially. Thus $\frac{5^{k}-1}{4}$ is not divisible by $2,5,7,17$ and 29 . This is not possible from $(3.7 b)$, since $\frac{5^{k}-1}{4} \mid \sigma^{* *}\left(5^{b}\right)$. This contradiction proves that $c=2, d=3$ is not admissible.

Let $c=2$ and $d=4$. We have $\sigma^{* *}\left(7^{4}\right)=2^{6} .43$. Taking $d=4$ in $(3.3 b)$, we find an imbalance in powers of 2 between both sides of (3.3b). Hence these values of $c$ and $d$ are not admissible.

The proof of part (b) of Theorem 3.2 is complete.

Proof of $(c)$. By hypothesis in this case $c=2$ and $d=1$ or 2 .

Let $c=2$ and $d=1$. By taking $d=1$ in $(3.3 a)$ and $(3.3 b)$, we get

$$
n=2^{7} \cdot 5^{b} \cdot 17^{2} \cdot 7 \cdot 29^{e} \cdot w^{\prime}
$$

and

$$
2^{3} \cdot 5^{b-2} \cdot 17 \cdot 7 \cdot 29^{e-1} \cdot w^{\prime}=\sigma^{* *}\left(5^{b}\right) \cdot \sigma^{* *}\left(29^{e}\right) \cdot \sigma^{* *}\left(w^{\prime}\right)
$$

where

$$
\left(w^{\prime}, 2.3 .5 .7 .17 .29\right)=1 \text { and } w^{\prime} \text { has no more than one odd prime factor, }
$$

where $b=2 k, k$ is odd and $\geq 3$. Also, $\sigma^{* *}\left(5^{b}\right)=\left(\frac{5^{k}-1}{4}\right) \cdot\left(5^{k+1}+1\right)$.

The factor $\frac{5^{k}-1}{4}>1$ is odd and is not divisible by 7,17 and 29 , since $k$ is odd and $\geq 3$; it is not divisible by 5 , trivially. Thus $\frac{5^{k}-1}{4}$ is not divisible by $2,5,7,17$ and 29 . Since $\frac{5^{k}-1}{4}>1$, let $p^{\prime} \mid \frac{5^{k}-1}{4}$. Then $p^{\prime}$ is odd and from $(3.8 b), p^{\prime} \mid w^{\prime}$.

Consider the factor $5^{k+1}+1$. We have (i) $2 \| 5^{k+1}+1$. (ii) $5^{k+1}+1$ is not divisible by 7 or 29 , since $k+1$ is even. (iii) Suppose $17 \mid 5^{k+1}+1$. Then $k+1=8 u$. Hence $5^{8}+1 \mid 5^{k+1}+1$. Also, $5^{8}+1=2.17 .11489$. It follows that $11489 \mid 5^{k+1}+1$. From $(3.8 b)$, we have $11489 \mid w^{\prime}$. Already $p^{\prime} \mid w^{\prime}$. Thus $w^{\prime}$ is divisible by two odd primes $p^{\prime}$ and 11489. This contradicts (3.8c) and so $17 \nmid 5^{k+1}+1$.

From (i)-(iii), it follows that $\frac{5^{k+1}+1}{2}>1$, is odd and not divisible by $5,7,17$ and 29 . Let $q^{\prime} \mid \frac{5^{k+1}+1}{2}$. Then from $(3.8 b), q^{\prime} \mid w^{\prime}$.

Thus $w^{\prime}$ is divisible by two distinct odd primes $p^{\prime}$ and $q^{\prime}$. This contradicts (3.8c).

Hence the case $c=2, d=1$ is not admissible.

Let $c=2$ and $d=2$. We have $\sigma^{* *}\left(7^{2}\right)=50=2.5^{2}$. Taking $(d=2)$, in $(3.3 a)$ and $(3.3 b)$, 
we get

$$
n=2^{7} \cdot 5^{b} \cdot 17^{2} \cdot 7^{2} \cdot 29^{e} \cdot w^{\prime}
$$

and

$$
2^{5} \cdot 5^{b-4} \cdot 17 \cdot 7^{2} \cdot 29^{e-1} \cdot w^{\prime}=\sigma^{* *}\left(5^{b}\right) \cdot \sigma^{* *}\left(29^{e}\right) \cdot \sigma^{* *}\left(w^{\prime}\right)
$$

where

$$
\left(w^{\prime}, 2.3 .5 .7 .17 .29\right)=1 \text { and } w^{\prime} \text { has no more than three odd prime factors. }
$$

In $(3.9 a)$ and $(3.9 b)$, we can assume that $b=2 k$ where $k$ is odd and $\geq 3$. We have

$$
\sigma^{* *}\left(5^{b}\right)=\left(\frac{5^{k}-1}{4}\right) \cdot\left(5^{k+1}+1\right)
$$

We prove that

(I) there exists an odd prime $p^{\prime}\left|\frac{5^{k}-1}{4}, p^{\prime}\right| w^{\prime}$ and $p^{\prime}>67$,

(II) there exists an odd prime $q^{\prime}\left|\frac{5^{k+1}+1}{2}, q^{\prime}\right| w^{\prime}$ and $q^{\prime}>67$.

Proof of (I). Let

$$
S_{5}^{\prime}=\left\{p \mid 5^{k}-1: p \in[3,67]-\{11,19,31\} \text { and } \operatorname{ord}_{p} 5 \text { is odd }\right\}
$$

By Lemma 2.3(a) (by replacing the interval $[3,2520]$ by $[3,67]$ ), if $S_{5}^{\prime}$ is non-empty, then (I) holds. Also, $11 \mid 5^{k}-1$ if and only if $5 \mid k$. Further, $5^{5}-1=2^{2} .11 .71$. Hence if $11 \mid 5^{k}-1$, then $71 \mid 5^{k}-1$. Thus $p^{\prime}=71\left|w^{\prime}, p^{\prime}\right| \frac{5^{k}-1}{4}$ and $p^{\prime}>67$. Thus (I) holds. In a similar manner, $19\left|5^{k}-1 \Longleftrightarrow 9\right| k$. Also, 829| $\frac{5^{9}-1}{4} \mid \frac{5^{k}-1}{4}$. Thus if $19 \mid 5^{k}-1$, then $p^{\prime}=829\left|\frac{5^{k}-1}{4}, p^{\prime}\right| w^{\prime}$ and $p^{\prime}>67$. Hence (I) holds in this case. It follows that if

$$
S_{5}^{\prime \prime}=\left\{p \mid 5^{k}-1: p \in[3,67]-\{31\} \text { and } \operatorname{ord}_{p} 5 \text { is odd }\right\},
$$

then $S_{5}^{\prime \prime}$ is non-empty implies that (I) holds.

Assume that $S_{5}^{\prime \prime}$ is empty. Since $5^{k}-1$ is not divisible by $p$ if $\operatorname{ord}_{p} 5$ is even, it follows that $5^{k}-1$ is not divisible by any prime in $[3,67]$ except for possibly 31 .

If $31 \nmid 5^{k}-1$, it follows that $5^{k}-1$ is not divisible by any prime in $[3,67]$. The same is true with respect to $\frac{5^{k}-1}{4}$ which is odd and $>1$. Let $p^{\prime} \mid \frac{5^{k}-1}{4}$. Then $p^{\prime} \notin[3,67]$ and so $p^{\prime}>67$. Also, from (3.9b), $p^{\prime} \mid w^{\prime}$. This proves (I) in this case.

We assume that $31 \mid 5^{k}-1$. We claim that $\frac{5^{k}-1}{4}$ is divisible by a prime $p^{\prime} \neq 31$. If this is not so, then we must have $\frac{5^{k}-1}{4}=31^{\alpha}$ for some positive integer $\alpha$. If $\alpha \geq 2$, then $31^{2} \mid 5^{k}-1$. Hence $93 \mid k$. In particular 31|k. Hence $1861\left|5^{31}-1\right| 5^{k}-1$. Thus $1861 \mid \frac{5^{k}-1}{4}=31^{\alpha}$. This is not possible. Hence $\alpha=1$ and so $\frac{5^{k}-1}{4}=31$ or $k=3$ or $b=6$.

We prove that $b=6$ is not admissible. Since $\sigma^{* *}\left(5^{8}\right)=2.31 .313$, taking $b=6$ in $(3.9 b)$, it follows that $w^{\prime}$ is divisible by 31 and 313. Let $w^{\prime}=31^{f} .313^{g} . w^{\prime \prime}$ Hence from $(3.9 a), n=$ $2^{7} .5^{b} \cdot 17^{2} \cdot 7^{2} \cdot 29^{e} .31^{f} .313^{g} . w^{\prime \prime}$, where $w^{\prime \prime}$ is 1 or a prime power. Let $w^{\prime \prime}=p^{h}$, where $p \geq 11$. 
We have

$$
3=\frac{\sigma^{* *}(n)}{n}<\frac{255}{128} \cdot \frac{19406}{15625} \cdot \frac{290}{289} \cdot \frac{50}{49} \cdot \frac{29}{28} \cdot \frac{31}{30} \cdot \frac{313}{312} \cdot \frac{11}{10}=2.992148375<3
$$

a contradiction. This proves that $b=6$ is not admissible.

Thus if $31 \mid 5^{k}-1$, then $\frac{5^{k}-1}{4}$ is divisible by a prime $p^{\prime} \neq 31$. It follows that $p^{\prime} \notin[3,67]$ and $p^{\prime} \mid w^{\prime}$ from $(3.9 b)$.

The proof of (I) is complete.

Proof of (II). Let

$$
T_{5}^{\prime}=\left\{q \mid 5^{k+1}+1: q \in[3,67]-\{13\} \text { and } s=\frac{1}{2} \operatorname{ord}_{q} 5 \text { is even }\right\} .
$$

In Lemma 2.3(b), if we replace the interval $[3,2520]$ by $[3,67]$, it follows that (II) holds whenever $T_{5}^{\prime}$ is non-empty.

Let $T_{5}^{\prime}$ be empty. Since $q \nmid 5^{k+1}+1$ if $s=\frac{1}{2} \operatorname{ord}_{q} 5$ is not even, it follows that $5^{k+1}+1$ is not divisible by any prime in $[3,67]$ except for possible 13 .

Suppose $13 \nmid 5^{k+1}+1$. Then $\frac{5^{k+1}+1}{2}>1$, is odd and is not divisible by any prime in $[3,67]$. If $q^{\prime} \mid \frac{5^{k+1}+1}{2}$, then $q^{\prime}>67$ and $q^{\prime} \mid w^{\prime}$ by $(3.9 b)$. Thus (II) holds in this case.

Suppose that $13 \mid 5^{k+1}+1$. We claim that $\frac{5^{k+1}+1}{2}$ is divisible by an odd prime $q^{\prime} \neq 13$. On the other hand, assume that $\frac{5^{k+1}+1}{2}=13^{\alpha}$, for some positive integer $\alpha$. If $\alpha \geq 2$, then $13^{2} \mid 5^{k+1}+1$. This is equivalent to $k+1=26 u$. Hence $5^{26}+1 \mid 5^{k+1}+1$, and so $53\left|\frac{5^{26}+1}{2}\right| \frac{5^{k+1}+1}{2}=13^{\alpha}$. This is not possible. Hence $\alpha=1$ so that $\frac{5^{k+1}+1}{2}=13$ or $k=1$. But $k \geq 3$. Hence we can find an odd prime $q^{\prime} \mid \frac{5^{k+1}+1}{2}$ and $q^{\prime} \neq 13$. It now follows that $q^{\prime} \notin[3,67]$. Also, from $(3.9 b), q^{\prime} \mid w^{\prime}$.

The proof of (II) is complete.

Since $w^{\prime}$ is divisible by $p^{\prime}, q^{\prime}$ and $p^{\prime} \neq q^{\prime}$, we can assume that $p^{\prime} \geq 71$ and $q^{\prime} \geq 73$. If $r$ denotes the possible third prime factor of $w^{\prime}$, we can assume that $r \geq 11$. From $(3.9 c)$, $w^{\prime}=\left(p^{\prime}\right)^{f} \cdot\left(q^{\prime}\right)^{g} \cdot r^{h}$. From $(3.9 a)$, we have $n=2^{7} \cdot 5^{b} \cdot 17^{2} \cdot 7^{2} \cdot 29^{e} \cdot\left(p^{\prime}\right)^{f} \cdot\left(q^{\prime}\right)^{g} \cdot r^{h}$. Hence

$$
3=\frac{\sigma^{* *}(n)}{n}<\frac{255}{128} \cdot \frac{5}{4} \cdot \frac{290}{289} \cdot \frac{50}{49} \cdot \frac{29}{28} \cdot \frac{71}{70} \cdot \frac{73}{72} \cdot \frac{11}{10}=2.98742924<3,
$$

a contradiction.

The case $c=2, d=2$ is complete. The proof of Theorem 3.2(c) is complete.

Proof of $(d)$. By hypothesis $c \geq 3$ and $d=1$. Since $c$ is even and $4 \nmid c$, we may assume that $c \geq 5$. By Lemma 2.1, we have $\frac{\sigma^{* *}\left(17^{c}\right)}{17^{c}} \geq \frac{25641254}{24137569} \quad(c \geq 5)$. From $(3.2 a)$, we have

$$
3=\frac{\sigma^{* *}(n)}{n} \geq \frac{255}{128} \cdot \frac{19406}{15625} \cdot \frac{25641254}{24137569} \cdot \frac{8}{7}=3.003889074>3
$$

a contradiction.

This completes the proof of (d). 
Proof of $(e)$. By hypothesis, $c \geq 3$ and $d=2$. Taking $d=2$ in $(3.2 a)$ and $(3.2 b)$, we get

$$
n=2^{7} \cdot 5^{b} \cdot 17^{c} \cdot 7^{2} \cdot w
$$

and

$$
2^{6} \cdot 5^{b-3} \cdot 17^{c-1} \cdot 7^{2} \cdot w=\sigma^{* *}\left(5^{b}\right) \cdot \sigma^{* *}\left(17^{c}\right) \cdot \sigma^{* *}(w),
$$

and

$$
(w, 2.3 .5 .7 .11 .13 .17)=1 \text { and } w \text { has no more than four odd prime factors. }
$$

By Remark 3.1, we can assume that $b=2 k$, where $k$ is odd and $\geq 3$. Also, $c=2 \ell$, where $\ell$ is odd and $\geq 3$, since $c \geq 3$.

We have

$$
\sigma^{* *}\left(5^{b}\right)=\left(\frac{5^{k}-1}{4}\right) \cdot\left(5^{k+1}+1\right)
$$

We prove that

(C) there exists an odd prime $p^{\prime}\left|\frac{5^{k}-1}{4}, p^{\prime}\right| w$ and $p^{\prime} \geq 2521$,

(D) there exists an odd prime $q^{\prime}\left|\frac{5^{k+1}+1}{2}, q^{\prime}\right| w$ and $q^{\prime} \geq 163$.

First we prove (D).

Proof of (D). Let

$$
T_{5}^{\prime}=\left\{q \mid 5^{k+1}+1: q \in[3,157]-\{13\} \text { and } s=\frac{1}{2} \operatorname{ord}_{q} 5 \text { is even }\right\} .
$$

Applying Lemma 2.3(b) (replacing the interval $[3,2520]$ by $[3,157]$ ) we conclude that if $T_{5}^{\prime}$ is non-empty, then (D) holds.

Assume that $T_{5}^{\prime}$ is empty. Since $q \nmid 5^{k+1}+1$ when $s=\frac{1}{2} \operatorname{ord}_{q} 5$ is not even, it follows that $\frac{5^{k+1}+1}{2}$ is not divisible by any prime $q$ in $[3,157]$ except for possibly $q=13$. Since (by hypothesis) $13 \nmid n$, it follows that $13 \nmid 5^{k+1}+1$ and thus $\frac{5^{k+1}+1}{2}$ is not divisible by any prime in $[3,157]$. Let $q^{\prime} \mid \frac{5^{k+1}+1}{2}$. Then $q^{\prime}>157$ (and so $q^{\prime} \geq 163$ ) and $q^{\prime} \mid w$.

This proves (D).

Proof of $(C)$. Let

$$
S_{5}=\left\{p \mid 5^{k}-1: p \in[3,2520]-\{11,19,31,71,181,829,1741\} \text { and } \operatorname{ord}_{p} 5 \text { is odd }\right\}
$$

If $S_{5}$ is non-empty, then (C) holds.

Let $S_{5}$ be empty. Since $p \nmid 5^{k}-1$ if $\operatorname{ord}_{p} 5$ is even, it follows that $\frac{5^{k}-1}{4}$ is not divisible by any prime $p \in[3,2520]$ except for possibly $p \in\{11,19,31,71,181,829,1741\}$.

By hypothesis, $11 \nmid n$. Hence $11 \nmid 5^{k}-1$; also, $11\left|5^{k}-1 \Longleftrightarrow 71\right| 5^{k}-1$. Hence $71 \nmid 5^{k}-1$.

Further, $181 \mid 5^{k}-1$ if and only if $15 \mid k$. Hence $181 \mid 5^{k}-1$ implies $11\left|5^{15}-1\right| 5^{k}-1$. But $11 \nmid 5^{k}-1$. Hence $181 \nmid 5^{k}-1$. Since $181\left|5^{k}-1 \Longleftrightarrow 1741\right| 5^{k}-1$, it follows that $1741 \nmid 5^{k}-1$.

We may note that $19\left|5^{k}-1 \Longleftrightarrow 9\right| k \Longleftrightarrow 829 \mid 5^{k}-1$. Suppose $19 \mid 5^{k}-1$. Hence $9 \mid k$ and consequently $5^{9}-1 \mid 5^{k}-1$. Also, $5^{9}-1=2^{2} .19 .31 .829$. Hence $\frac{5^{k}-1}{4}$ and consequently $\sigma^{* *}\left(5^{b}\right)$ 
is divisible by 19,31 and 829 . From, (3.10b), it follows that $w$ is divisible by 19,31 and 829 . Hence $w=19^{e} .31^{f} .(829)^{g} . w^{\prime}$, so that from $(3.10 a)$ and $(3.10 b)$, we have

$$
n=2^{7} \cdot 5^{b} \cdot 17^{c} \cdot 7^{2} \cdot 19^{e} \cdot 31^{f} \cdot 829^{g} \cdot w^{\prime}
$$

and

$$
2^{6} .5^{b-3} .17^{c-1} .7^{2} .19^{e} .31^{f} .829^{g} . w^{\prime}=\sigma^{* *}\left(5^{b}\right) \sigma^{* *}\left(17^{c}\right) \sigma^{* *}\left(19^{e}\right) \sigma^{* *}\left(31^{f}\right) \sigma^{* *}\left(829^{g}\right) \sigma^{* *}\left(w^{\prime}\right),
$$

and

$\left(w^{\prime}, 2.3 .5 .7 .11 .13 .17 .19 .31 .829\right)=1$ and $w^{\prime}$ has no more than one odd prime factor. $(3.11 c)$

By what we have proved in (D), $q^{\prime} \mid w$ and $q^{\prime}>157$ (that is, $q^{\prime} \geq 163$ ). By $(3.11 c), w^{\prime}=\left(q^{\prime}\right)^{h}$. Hence $n=2^{7} .5^{b} .17^{c} .7^{2} .19^{e} .31^{f} .(829)^{g} .\left(q^{\prime}\right)^{h}$, so that by Lemma 2.1,

$$
3=\frac{\sigma^{* *}(n)}{n}<\frac{255}{128} \cdot \frac{5}{4} \cdot \frac{17}{16} \cdot \frac{50}{49} \cdot \frac{19}{18} \cdot \frac{31}{30} \cdot \frac{829}{828} \cdot \frac{163}{162}=2.966616483<3
$$

a contradiction.

Hence 19 and consequently 829 cannot divide $5^{k}-1$.

Till now, $\frac{5^{k}-1}{4}>1$, is odd and is not divisible by any prime in [3, 2520] except for possibly by 31 . If $31 \nmid 5^{k}-1$, then $\frac{5^{k}-1}{4}(>1)$ will not be divisible by any prime in [3, 2520]. If $p^{\prime} \mid \frac{5^{k}-1}{4}$, then $p^{\prime} \notin[3,2520]$ and $p^{\prime} \mid w$. This would prove (C).

Suppose that $31 \mid 5^{k}-1$. We claim that we can find an odd prime $p^{\prime} \mid \frac{5^{k}-1}{4}$ and $p^{\prime} \neq 31$. If this is not so, then we must have $\frac{5^{k}-1}{4}=31^{\alpha}$, where $\alpha$ is a positive integer. If $\alpha \geq 2$, then $31^{2} \mid 5^{k}-1$; this is equivalent to $93 \mid k$. In particular, $31 \mid k$ and so $5^{31}-1 \mid 5^{k}-1$. Thus $1861\left|\frac{5^{31}-1}{4}\right| \frac{5^{k}-1}{4}=31^{\alpha}$. This is impossible. Hence $\alpha=1$ and consequently $\frac{5^{k}-1}{4}=31$ or $k=3$ or $b=6$.

We now show that $b=6$ is not possible. We have $\sigma^{* *}\left(5^{6}\right)=2.31 .313$. Taking $b=6$ in $(3.10 b)$, we find that 31 and 313 are factors of $w$. Let $w=31^{e} .313^{f} . w^{\prime}$. Now, from $(3.10 a)$ and $(3.10 b)$, we have

$$
n=2^{7} \cdot 5^{b} \cdot 17^{c} \cdot 7^{2} \cdot 31^{e} \cdot 313^{f} \cdot w^{\prime},
$$

and

$$
2^{5} \cdot 5^{b-3} \cdot 17^{c-1} \cdot 7^{2} \cdot 31^{e-1} \cdot 313^{f-1} \cdot w^{\prime}=\sigma^{* *}\left(17^{c}\right) \cdot \sigma^{* *}\left(31^{e}\right) \cdot \sigma^{* *}\left(313^{f}\right) \cdot \sigma^{* *}\left(w^{\prime}\right),
$$

and

$\left(w^{\prime}, 2.3 .5 .7 .11 .13 .17 .31 .313\right)=1$ and $w^{\prime}$ has no more than two odd prime factors.

From Remark 3.1, we can assume that $c=2 \ell$, where $\ell$ is odd and $\geq 3$ (since $c \geq 3$ ). We have $\sigma^{* *}\left(17^{c}\right)=\left(\frac{17^{\ell}-1}{16}\right) \cdot\left(17^{\ell}+1\right)$. We obtain a contradiction by showing that

(E) $\frac{17^{\ell}-1}{16}$ is divisible by an odd prime $p^{\prime}>127$. 
Let

$$
S_{17}=\left\{p \mid 17^{\ell}-1: p \in[3,127] \text { and } \operatorname{ord}_{p} 17 \text { is odd }\right\} .
$$

By Lemma 2.6(a), if $S_{17}$ is non-empty, then we can find an odd prime $p^{\prime} \mid \frac{17^{\ell}-1}{16}$ and $p^{\prime}>127$. Hence (E) follows in this case.

Suppose that $S_{17}$ is empty. Since $p \nmid 17^{\ell}-1$ if $\operatorname{ord}_{p} 17$ is even, it follows that $17^{\ell}-1$ is not divisible by any prime in $[3,127]$. Since $\frac{17^{\ell}-1}{16}>1$, is odd and is not divisible by any prime in $[3,127]$, if $p^{\prime} \mid \frac{17^{\ell}-1}{16}$, then $p^{\prime}>127$ or $p^{\prime} \geq 131$. Further, since $313\left|17^{\ell}-1 \Longleftrightarrow 312\right| \ell$, it follows that $313 \nmid 17^{\ell}-1$ since $\ell$ is odd. Hence $p^{\prime} \neq 313$. From $(3.12 b)$, it is clear that $p^{\prime} \mid w^{\prime}$. By $(3.12 c), w^{\prime}$ has no more than two odd prime factors. If $r \neq p^{\prime}$ denotes the possible second prime factor of $w^{\prime}$, then $r \geq 19$. From $(3.12 a)$, we have $n=2^{7} \cdot 5^{b} \cdot 17^{c} \cdot 7^{2} \cdot 31^{e} \cdot 313^{f} \cdot\left(p^{\prime}\right)^{g} \cdot r^{h}$ so that by Lemma 2.1,

$$
3=\frac{\sigma^{* *}(n)}{n}<\frac{255}{128} \cdot \frac{5}{4} \cdot \frac{17}{16} \cdot \frac{50}{49} \cdot \frac{31}{30} \cdot \frac{313}{312} \cdot \frac{131}{130} \frac{19}{18}=2.977023814<3,
$$

a contradiction. Hence (E) holds.

We thus finally proved that $b=6$ is not admissible. This means that $\frac{5^{k}-1}{4}$ is divisible by an odd prime $p^{\prime} \neq 31$. Also, as $\frac{5^{k}-1}{4}$ is not divisible by any prime in $[3,2520]-\{31\}$, it now follows that $p^{\prime} \notin[3,2520]$. Also, $p^{\prime} \mid w$ by $(3.10 b)$. This proves $(\mathbf{C})$.

By $(3.10 c), w$ has no more than four odd prime factors; $p^{\prime}$ and $q^{\prime}$ are two prime factors of $w$. Let the other two possible prime factors be $r$ and $t$. We can assume that $r \geq 19$ and $s \geq 23$. From $(3.10 a)$, we have $n=2^{7} \cdot 5^{b} \cdot 17^{c} \cdot 7^{2} \cdot\left(p^{\prime}\right)^{e} \cdot\left(q^{\prime}\right)^{f} \cdot r^{f} \cdot t^{h}$ and so by Lemma 2.1,

$$
3=\frac{\sigma^{* *}(n)}{n}<\frac{255}{128} \cdot \frac{5}{4} \cdot \frac{17}{16} \cdot \frac{50}{49} \cdot \frac{2521}{2520} \cdot \frac{163}{162} \cdot \frac{19}{18} \cdot \frac{23}{22}=2.998984579<3,
$$

a contradiction.

This completes the proof of Theorem 3.2 (e).

Theorem 3.3. Let $n$ be as given in (3.1a), where $b \geq 3$ and $3 \nmid n$. Assume that $7^{2} \| n$ and $17^{3} \mid n$. Let $n$ be a bi-unitary triperfect number. Let $s=11$ or $s=13$. Assume that $s \mid n$, so that from (3.10b), $w=s^{e} . w^{\prime}$ and consequently from (3.10a),

$$
n=2^{7} \cdot 5^{b} \cdot 17^{c} \cdot 7^{2} \cdot s^{e} \cdot w^{\prime} .
$$

Then

(a) $c=6$ is not admissible.

If $c=2 \ell$, where $\ell$ is odd, then

(b) $\frac{17^{\ell}-1}{16}$ is divisible by an odd prime $p^{\prime}>519$ and $p^{\prime} \mid w^{\prime}$,

(c) $\frac{17^{\epsilon}+1}{2}$ is divisible by an odd prime $q^{\prime}>519$ and $q^{\prime} \mid w^{\prime}$.

Proof. Substituting $w=s^{e} \cdot w^{\prime}$ in $(3.10 b)$, we obtain

$$
2^{6} \cdot 5^{b-3} \cdot 17^{c-1} \cdot 7^{2} \cdot s^{e} \cdot w^{\prime}=\sigma^{* *}\left(5^{b}\right) \cdot \sigma^{* *}\left(17^{c}\right) \cdot \sigma^{* *}\left(s^{e}\right) \cdot \sigma^{* *}\left(w^{\prime}\right)
$$


also,

$$
\left(w^{\prime}, 2 \text {.3.5.7.s.17) }=1 \text { and } w^{\prime}\right. \text { has no more than three odd prime factors. }
$$

By Remark 3.1, we can assume that $b=2 k$, where $k$ is odd and $\geq 3$. Also, $c=2 \ell$, where $\ell$ is odd and $\geq 3$ since $c \geq 3$.

Proof of $(a)$. We have $\sigma^{* *}\left(17^{6}\right)=2.307 .41761$. Hence taking $c=6$ in $(3.13 b)$, we obtain

$$
2^{5} \cdot 5^{b-3} \cdot 17^{5} \cdot 7^{2} \cdot s^{e} \cdot w^{\prime}=307 \cdot 41761 \cdot \sigma^{* *}\left(5^{b}\right) \cdot \sigma^{* *}\left(s^{e}\right) \cdot \sigma^{* *}\left(w^{\prime}\right)
$$

From $(3.13 d), w^{\prime}$ is divisible by 307 and 41761 . Hence we may assume that

$$
w^{\prime}=(307)^{f} \cdot(41761)^{g} \cdot w^{\prime \prime}
$$

using this in $(3.13 a)$ and $(3.13 d)$, we get

$$
n=2^{7} \cdot 5^{b} \cdot 17^{6} \cdot 7^{2} \cdot s^{e} \cdot(307)^{f} \cdot(41761)^{g} \cdot w^{\prime \prime},
$$

and

$$
\begin{gathered}
2^{5} \cdot 5^{b-3} \cdot 17^{5} \cdot 7^{2} \cdot s^{e} \cdot(307)^{f-1} \cdot(41761)^{g-1} \cdot w^{\prime \prime} \\
=\sigma^{* *}\left(5^{b}\right) \cdot \sigma^{* *}\left(s^{e}\right) \cdot \sigma^{* *}\left((307)^{f}\right) \cdot \sigma^{* *}\left((41761)^{g}\right) \sigma^{* *}\left(w^{\prime \prime}\right),
\end{gathered}
$$

where

$$
\left(w^{\prime \prime}, 2.3 .5 .7 . s .17 .307 .41761\right)=1 \text { and } w^{\prime \prime} \text { is } 1 \text { or a prime power. }
$$

We obtain a contradiction by examining the factors of $\sigma^{* *}\left(5^{b}\right)$ as follows.

We have $b=2 k$, where $k$ is odd and $\geq 3$. Also, $\sigma^{* *}\left(5^{b}\right)=\left(\frac{5^{k}-1}{4}\right) \cdot\left(5^{k+1}+1\right)$. We claim that we can find two distinct odd primes $p$ and $q$ such that

(I) $p \mid \frac{5^{k}-1}{4}$ and $p \mid w^{\prime \prime}$ and (II) $q \mid \frac{5^{k+1}+1}{2}$ and $q \mid w^{\prime \prime}$.

- Proof of (I).

(i) Since $k$ is odd, we have $4 \| 5^{k}-1$. Hence $\frac{5^{k}-1}{4}$ is odd; also, it is $>1$, since $k \geq 3$.

(ii) We have $7\left|5^{t}-1 \Longleftrightarrow 6\right| t ; 17\left|5^{t}-1 \Longleftrightarrow 16\right| t ; 13\left|5^{t}-1 \Longleftrightarrow 4\right| t ; 307 \mid 5^{t}-1 \Longleftrightarrow$ $306 \mid t$ and $41761\left|5^{t}-1 \Longleftrightarrow 4176\right| t$. In all these cases, first of all $t$ must be even. Since $k$ is odd, $5^{k}-1$ is not divisible by any of the primes in $\{7,13,17,307,41761\}$; and is not divisible by 5 trivially.

(iii) We have $11\left|5^{k}-1 \Longleftrightarrow 5\right| k \Longleftrightarrow 71 \mid 5^{k}-1$. Hence if $11 \mid 5^{k}-1$ from $(3.14 b)$, it follows that $71 \mid w^{\prime \prime}$. Hence (I) holds in this case. We may assume that $11 \nmid 5^{k}-1$. Then $\frac{5^{k}-1}{4}$ is odd, is $>1$ and is not divisible by $5,7,11,13,17,307$ and 41761 or it is not divisible by $5,7, s, 17,307$ and 41761 . It follows that if $p \mid \frac{5^{k}-1}{4}$, then $p \notin\{5,7, s, 17,307,41761\}$. From $(3.14 b)$, we conclude that $p \mid w^{\prime \prime}$.

This proves (I). 
- Proof of (II). Consider the factor $5^{k+1}+1$, where $k$ is odd and $\geq 3$.

(iv) $2 \| 5^{k+1}+1$ and so $\frac{5^{k+1}+1}{2}$ is odd and clearly $>1$.

(v) For any positive integer $t, 5^{t}+1$ is not divisible by 11 and trivially not divisible by 5. In particular, $5^{k+1}+1$ is not divisible by 5 and 11 .

(vi) $7\left|5^{k+1}+1 \Longleftrightarrow k+1=3 u ; 307\right| 5^{k+1}+1 \Longleftrightarrow k+1=153 u$. Since $k+1$ is even $5^{k+1}+1$ is not divisible by 7 or 307 .

(vii) $17\left|5^{k+1}+1 \Longleftrightarrow k+1=8 u ; 41761\right| 5^{k+1}+1 \Longleftrightarrow k+1=2088 u=8 u^{\prime}$, where $u^{\prime}$ is odd. If either $17 \mid 5^{k+1}+1$ or $41761 \mid 5^{k+1}+1$, it follows that $5^{8}+1 \mid 5^{k+1}+1$. Also, $5^{8}+1=2.17 .11489$. Hence $11489 \mid 5^{k+1}+1$. From (3.14b), it follows that $11489 \mid w^{\prime \prime}$. In both the cases it follows that (II) holds with $q=11489$. In what follows, assume that $5^{k+1}+1$ is neither divisible by 17 nor 41761 .

(viii) Assume that $13 \nmid 5^{k+1}+1$. From (iv) to (vii), it follows that $\frac{5^{k+1}+1}{2}$ is not divisible by $5,7,11,13,17,307$ and 41761 ; in particular, it is not divisible by any of the primes $5,7, s, 17,307$ and 41761. Hence if $q \mid \frac{5^{k+1}+1}{2}$, then $q \notin\{5,7, s, 17,307,41761\}$. From (3.14b), $q \mid w^{\prime \prime}$. This proves (II) in this case.

(ix) Assume that $13 \mid 5^{k+1}+1$. Assume that $\frac{5^{k+1}+1}{2}$ is divisible by 13 alone so that $\frac{5^{k+1}+1}{2}=13^{\alpha}, \alpha$ being a positive integer. Suppose $\alpha \geq 2$. Hence $13^{2} \mid 5^{k+1}+1$; this holds if and only if $k+1=26 u$ and so $5^{26}+1 \mid 5^{k+1}+1$. Also, $5^{26}+$ $1=2.13^{2} .53 .83181652304609$. Thus $53\left|\frac{5^{26}+1}{2}\right| \frac{5^{k+1}+1}{2}=13^{\alpha}$, which is impossible. Hence $\alpha=1$ and so $\frac{5^{k+1}+1}{2}=13$ so that $k=1$. But $k \geq 3$. It follows that $\frac{5^{k+1}+1}{2}$ is divisible by an odd prime $q \neq 13$. Clearly, $q \notin\{5,7, s, 17,307,41761\}$. From $(3.14 b), q \mid w^{\prime \prime}$.

This proves (II).

Thus $p$ and $q$ are factors of $w^{\prime \prime}$. This violates $(3.14 c)$. This proves that $c=6$ is not admissible.

The proof of Theorem 3.3(a) is complete.

Proof of $(b)$. By (a), $c \neq 6$. Hence $\ell \geq 5$. Now from (I) of Corollary 2.1, it follows that $\frac{17^{\ell}-1}{16}$ is divisible by an odd prime $p^{\prime}>519$; that $p^{\prime} \mid w^{\prime}$ readily follows from $(3.13 b)$. Hence (b) follows.

Proof of $(c)$. By (II) of Corollary 2.1, it follows that $\frac{17^{\ell+1}+1}{2}$ is divisible by an odd prime

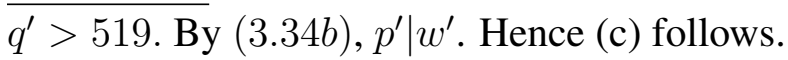

This completes the proof of Theorem 3.3.

Theorem 3.4. Let $n$ be as given in (3.1a), where $b \geq 3$ and $3 \nmid n$. Assume that $7^{2} \| n$ and $17^{3} \mid n$. Let $n$ be a bi-unitary triperfect number.

(a) Then $n$ is not divisible by 11 and 13 simultaneously.

(b) Suppose that $n$ is divisible by 11 or 13 . Let $s=11$ or 13 . Then we have

$$
n> \begin{cases}4.5349 \times 10^{169}, & \text { if } s=11 \\ 3.43 \times 10^{114}, & \text { if } s=13\end{cases}
$$


Proof. The relevant equations are (3.10a) and (3.10b).

Proof of $(a)$. We assume that $n$ is divisible by 11 and 13 . From $(3.10 a)$, we find that 11 and 13 divide $w$. Let $w=11^{e} .13^{f} . w^{\prime}$, where $\left(w^{\prime}, 2.3 .5 .7 .11 .13 .17\right)=1$. From $(3.10 a)$ and $(3.10 b)$, we obtain

$$
n=2^{7} \cdot 5^{b} \cdot 17^{c} \cdot 7^{2} \cdot 11^{e} \cdot 13^{f} \cdot w^{\prime}
$$

and

$$
2^{6} \cdot 5^{b-3} \cdot 17^{c-1} \cdot 7^{2} \cdot 11^{e} \cdot 13^{f} \cdot w^{\prime}=\sigma^{* *}\left(5^{b}\right) \cdot \sigma^{* *}\left(17^{c}\right) \cdot \sigma^{* *}\left(11^{e}\right) \cdot \sigma^{* *}\left(13^{f}\right) \cdot \sigma^{* *}\left(w^{\prime}\right),
$$

where

$$
\left(w^{\prime}, 2.3 .5 .7 .11 .13 .17\right)=1 \text { and } w^{\prime} \text { has no more than two odd prime factors. }
$$

We recall that $b=2 k$ and $c=2 \ell$, where $k, \ell$ are both $\geq 3$ and odd. We can take $b \geq 5$ and $c \geq 3$ by hypothesis. We have by Lemma 2.1 ,

$$
\begin{aligned}
\frac{\sigma^{* *}\left(5^{b}\right)}{5^{b}} \geq \frac{19406}{15625}, \quad(b \geq 5) ; & \frac{\sigma^{* *}\left(17^{c}\right)}{17^{c}} \geq \frac{88452}{83521}, \quad(c \geq 3) ; \\
\frac{\sigma^{* *}\left(11^{e}\right)}{11^{e}} \geq \frac{15984}{14641}, \quad(e \geq 3) ; & \frac{\sigma^{* *}\left(13^{f}\right)}{13^{f}} \geq \frac{30772}{28561}, \quad(f \geq 3) .
\end{aligned}
$$

Hence for $e \geq 3$ and $f \geq 3$, from $(3.15 a)$, we have

$$
3=\frac{\sigma^{* *}(n)}{n} \geq \frac{255}{128} \cdot \frac{19406}{15625} \cdot \frac{88452}{83521} \cdot \frac{50}{49} \cdot \frac{15984}{14641} \cdot \frac{30772}{28561}=3.145061575,
$$

a contradiction.

Thus $e \geq 3$ and $f \geq 3$ cannot hold. The following cases arise:

(i) $\{e \geq 3, f=1,2\}$ (ii) $\{e=1,2, f \geq 3\}$ and (iii) $\{e=1,2, f=1,2\}$.

(i) Let $e \geq 3$ and $f=1$. From $(3.15 a)$, we have

$$
3=\frac{\sigma^{* *}(n)}{n} \geq \frac{255}{128} \cdot \frac{19406}{15625} \cdot \frac{88452}{83521} \cdot \frac{50}{49} \cdot \frac{15984}{14641} \cdot \frac{14}{13}=3.143630701>3,
$$

a contradiction.

Let $f=2$. We will not be using that $e \geq 3$. By (b) and (c) of Theorem 3.3, $w^{\prime}$ is divisible by two distinct odd primes $p^{\prime}$ and $q^{\prime}$ exceeding 519. We may assume that $p^{\prime} \geq 521$ and $q^{\prime} \geq 523$. Also, by $(3.15 c), w^{\prime}=\left(p^{\prime}\right)^{g} \cdot\left(q^{\prime}\right)^{h}$. Hence from $(3.15 a)$, $n=2^{7} \cdot 5^{b} \cdot 17^{c} \cdot 7^{2} \cdot 11^{e} \cdot 13^{2} \cdot w^{\prime}$ and so we have

$$
3=\frac{\sigma^{* *}(n)}{n}<\frac{255}{128} \cdot \frac{5}{4} \cdot \frac{17}{16} \cdot \frac{50}{49} \cdot \frac{11}{10} \cdot \frac{170}{169} \cdot \frac{521}{520} \cdot \frac{523}{522}=2.998910842<3,
$$

a contradiction. 
(ii) Let $e=1$. Since $3 \mid 12=\sigma^{* *}(11)$, taking $e=1$ in $(3.15 b)$, it follows that 3 is a factor of its left-hand side. This cannot happen. Hence $e=1$ is not admissible (independent of $f \geq 3$ ).

Let $e=2$. We have $\sigma^{* *}\left(11^{2}\right)=122=2.61$. Taking $e=2$, in $(3.15 b)$, we find that $61 \mid w^{\prime}$. By (b) and (c) of Theorem 3.3, $w^{\prime}$ is already divisible by two odd primes $p^{\prime}$ and $q^{\prime}$ exceeding 61. Thus $w^{\prime}$ is divisible by three odd primes, namely, 61, $p^{\prime}$ and $q^{\prime}$. This cannot happen in view of $(3.15 c)$. Thus $e=2$ is not admissible (independent of $f \geq 3$ ). Thus (ii) cannot hold.

(iii) This case will not occur as neither $e=1$ nor $e=2$ is admissible.

This proves part (a) of Theorem 3.4.

Proof of $(b)$. We have $n=2^{7} \cdot 5^{b} \cdot 17^{c} \cdot 7^{2} \cdot s^{e} \cdot w^{\prime}$, and $n$ satisfies $(3.13 b)$ and $(3.13 c)$.

We prove that $b \geq 54$ and $c \geq 54$. We recall that $b=2 k$ and $c=2 \ell$, where $k$ and $\ell$ are odd and $\geq 3$.

(1) We have $\sigma^{* *}\left(5^{6}\right)=2.31 .313$. Taking $b=6$ in $(3.13 b)$, we see that $w^{\prime}$ is divisible by 31 and 313. By Theorem 3.3 (b) and (c), $w^{\prime}$ is divisible by $p^{\prime}$ and $q^{\prime}$ both exceeding 519. Hence $w^{\prime}$ must be divisible by four odd prime factors namely, 31, 313, $p^{\prime}$ and $q^{\prime}$. This contradicts $(3.13 c)$. Hence $b=6$ is not possible.

(2) We have $\sigma^{* *}\left(5^{10}\right)=2.11 .13 .71 .601$. Taking $b=10$ in $(3.13 b)$, we infer that $w^{\prime}$ is divisible by 11 and 13 . By (a) of the present theorem this is not possible. Hence $b=10$ is not admissible.

(3) Let $b=14$. We have $\sigma^{* *}\left(5^{14}\right)=2.17 .11489 .19531$; and $11489\left|17^{\ell}-1 \Longleftrightarrow 11488\right| \ell$. This is not possible since $\ell$ is odd. Hence $11489 \nmid 17^{\ell}-1$. Let $p=11489$ and $r=\operatorname{ord}_{p} 17$ so that $r=11488$. Hence $r / 2=5744=2^{4} .379$. Hence $p \mid 17^{\ell+1}+1$ if and only if $\ell+1=\frac{r}{2} \cdot u=16.379 . u=16 . u^{\prime}$, where $u^{\prime}$ is odd. Thus $p \mid 17^{\ell+1}+1$ implies that $17^{16}+1$ is a factor of $17^{\ell+1}+1$. We have

$$
17^{16}+1=\{\{2,1\},\{257,1\},\{1801601,1\},\{52548582913,1\}\} .
$$

It now follows from $(3.13 b)$ that $w^{\prime}$ is divisible by five odd prime factors, namely, 257 , $1801601,52548582913,11489$ and 19531. This contradicts $(3.13 c)$. Hence $p \nmid 17^{\ell+1}+1$ and so $p \nmid \sigma^{* *}\left(17^{c}\right)$.

Let $q=19531$. Then $r^{\prime}=\operatorname{ord}_{q} 17=9765=3^{2} \cdot 5.7 .31$. Hence $31 \mid r^{\prime}$ and so $17^{31}-$ $1 \mid 17^{\ell}-1$ if $q \mid 17^{\ell}-1$. Also,

$$
17^{31}-1=\{\{2,4\},\{4093,1\},\{6123493,1\},\{347340647626008901939025023,1\}\} .
$$

It now follows from $(3.13 b)$ that $w^{\prime}$ is divisible by five odd prime factors, namely, 11489, 19531, 4093, 6123493 and 347340647626008901939025023 . This contradicts $(3.13 c)$. Hence $q \nmid 17^{\ell}-1$. 
Since $r^{\prime}=9765$ is odd, $q \nmid 17^{t}+1$ for any positive integer $t$. In particular, $q \nmid 17^{\ell+1}+1$. Hence from the above discussion it follows that $q \nmid \sigma^{* *}\left(17^{c}\right)$. Let $p^{\prime}$ and $q^{\prime}$ be as given in (b) and (c) of Theorem 3.3. Then the four distinct primes $p, q, p^{\prime}$ and $q^{\prime}$ are factors of $w^{\prime}$ by $(3.13 b)$. This is a contradiction to $(3.13 c)$. Hence $b=14$ is not admissible.

(4) We have $\sigma^{* *}\left(5^{18}\right)=2.13 .19 .31 .41 .829 .9161$. Taking $b=18$ in $(3.13 b)$, we see that $w^{\prime}$ is divisible by five odd primes, namely, 19, 31, 41, 829 and 9161 . This violates $(3.13 c)$. Hence $b=18$ is not admissible.

(5) We have $\sigma^{* *}\left(5^{22}\right)=2.313 .390001 .12207031$. Taking $b=22$ in (3.13b), we find that $w^{\prime}=(313)^{f} \cdot(390001)^{g} \cdot(12207031)^{h}$. Hence from $(3.13 a)$,

$$
n=2^{7} \cdot 5^{b} \cdot 17^{c} \cdot 7^{2} \cdot s^{e} \cdot(313)^{f} \cdot(390001)^{g} \cdot(12207031)^{h},
$$

so that

$$
3=\frac{\sigma^{* *}(n)}{n}<\frac{255}{128} \cdot \frac{5}{4} \cdot \frac{17}{16} \cdot \frac{50}{49} \cdot \frac{11}{10} \cdot \frac{313}{312} \cdot \frac{390001}{390000} \cdot \frac{12207031}{12207030}=2.979385259<3,
$$

a contradiction. Hence $b=22$ is not admissible.

(6) Let $b=26$. We have $\sigma^{* *}\left(5^{26}\right)=\{\{2,1\},\{13,1\},\{234750601,1\},\{305175781,1\}\}$. Let $p=234750601$ and $q=305175781$. We now prove that $\sigma^{* *}\left(17^{c}\right)$ is not divisible by either $p$ or $q$. We have

$$
\sigma^{* *}\left(17^{c}\right)=\left(\frac{17^{\ell}-1}{16}\right) \cdot\left(17^{\ell+1}+1\right)
$$

Let $r=$ ord $_{p} 17$. Then $r \mid p-1=234750600=\{\{2,3\},\{3,2\},\{5,2\},\{7,1\},\{31,1\},\{601,1\}\}$ and hence $r$ takes 288 choices. Verifying these choices, we can show that $r=5868765$.

Since $45 \mid r$, we have $17^{45}-1 \mid 17^{r}-1$. Assume that $p \mid 17^{\ell}-1$. Hence $r \mid \ell$ so that $17^{45}-$ $1\left|17^{r}-1\right| 17^{\ell}-1$. Also,

$$
\begin{aligned}
17^{45}-1=\{ & \{2,4\},\{19,1\},\{307,1\},\{3691,1\},\{33931,1\},\{88741,1\},\{316531,1\}, \\
& \{1270657,1\},\{1674271,1\},\{5113320301,1\},\{6566760001,1\}\} .
\end{aligned}
$$

It follows from $(3.13 b)$ that $w^{\prime}$ will be divisible by ten odd prime factors. This violates $(3.13 c)$. Thus $p \nmid 17^{\ell}-1$. Since $r$ is odd, $p \nmid 17^{t}+1$ for any positive integer $t$. In particular, $p \nmid 17^{\ell+1}+1$.

Let $r^{\prime}=$ ord $d_{q} 17$. Then $r^{\prime} \mid q-1=305175180=\{\{2,2\},\{3,1\},\{5,1\},\{367,1\},\{13859,1\}\}$. Hence $r^{\prime}$ takes 48 choices. Verifying these choices it can be shown that $r^{\prime}=21798270$. As $r^{\prime}$ is even and $\ell$ is odd, $q \nmid 17^{\ell}-1$. Also, if $s^{\prime}=r^{\prime} / 2=10899135$, then $s^{\prime}$ is odd. Hence $q \nmid 17^{\ell+1}+1$ since $\ell+1$ is even.

It follows that $\sigma^{* *}\left(17^{c}\right)$ is not divisible by either $p$ or $q$. If $p^{\prime}$ and $q^{\prime}$ are as in (b) and (c) of Theorem 3.3, it is clear from (3.13b) that $p, p^{\prime}, q$ and $q^{\prime}$ are four distinct odd prime factors of $w^{\prime}$. This is a contradiction to (3.13c). Thus $b=26$ is not admissible. 
(7) Since $\sigma^{* *}\left(5^{30}\right)=2.11 .31 .71 .181 .1741 .2593 .29423041$, taking $b=30$ in (3.13b), we infer that $w^{\prime}$ will be divisible by six odd prime factors and this violates $(3.13 c)$. Hence $b=30$ is not admissible.

In a similar way we have

$$
\begin{aligned}
& \sigma^{* *}\left(5^{34}\right)=2.13 .37 .409 .601 .6597973 .466344409, \\
& \sigma^{* *}\left(5^{38}\right)=2.191 .241 .313 .6271 .3981071 .632133361, \\
& \sigma^{* *}\left(5^{42}\right)=2.13 .31 .89 .379 .19531 .519499 .1030330938209, \\
& \sigma^{* *}\left(5^{46}\right)=2.17 .8971 .11489 .152587500001 .332207361361, \\
& \sigma^{* *}\left(5^{50}\right)=2.11 .13 .53 .71 .101 .251 .401 .9384251 .83181652304609 .
\end{aligned}
$$

Hence if $b=34,38,42,46$ or 50 , from $(3.13 b)$, we infer that $(3.13 c)$ is violated. Hence $b \geq 54$.

We now show that $c \geq 56$.

(8) We recall that $c=2 \ell$, where $\ell$ is odd and $\geq 3$. By (a) of Theorem $3.3, c=6$ is not admissible.

(9) Let $c=10$. We have $\sigma^{* *}\left(17^{10}\right)=2.5 .29 .83233 .88741$. Let $p=83233$ and $q=88741$. Then $r=\operatorname{ord}_{p} 5=9248$. Since $r$ is even, $p \nmid 5^{k}-1$. Let $s^{\prime}=r / 2=4624$. Suppose that $p \mid 5^{k+1}+1$. This is equivalent to $k+1=s^{\prime} u=(4624) u=16 u^{\prime}$, where $u^{\prime}$ is odd. Hence $5^{16}+1 \mid 5^{k+1}+1$. Also, $5^{16}+1=2.2593 .29423041$. From (3.13b), we infer that $w^{\prime}$ will be divisible by the five odd primes $2593,29423041,29,83233$ and 88741 . This is not possible in virtue of $(3.13 c)$. Hence $p \nmid 5^{k+1}+1$.

We have $r^{\prime}=\operatorname{ord}_{q} 5=44370$. Since $r^{\prime}$ is even, $q \nmid 5^{k}-1$. Also, $s^{\prime}=r^{\prime} / 2=22185$ and so $s^{\prime}$ is odd. Hence $q \nmid 5^{k+1}+1$.

Thus neither $p$ nor $q$ divides $\sigma^{* *}\left(5^{b}\right)$.

We now prove that we can find a prime $t \mid \frac{5^{k}-1}{4}$ and $t \notin\{5,7,11,13,29\}$. Since $k$ is odd, $5^{k}-1$ is not divisible by 7,13 and 29 . Suppose that $11 \nmid 5^{k}-1$. If $t$ is any prime factor of $\frac{5^{k}-1}{4}$ which is $>1$ and odd, then $t \notin\{5,7,11,13,29\}$. Suppose that $11 \mid 5^{k}-1$. This is equivalent to $71 \mid 5^{k}-1$. Hence we can take $t=71$. From $(3.13 b)$, in both the cases $t \mid w^{\prime}$ and $t \notin\{p, q\}$. Hence 29, $t, p, q$ would be four prime factors of $w^{\prime}$ in $(3.13 b)$ and this violates $(3.13 c)$.

Thus $c=10$ is not admissible.

(10) We have $\sigma^{* *}\left(17^{14}\right)=2.18913 .184417 .25646167$. Taking $c=14$ in (3.13b), we find that $w^{\prime}=(18913)^{f} \cdot(184417)^{g} \cdot(25646167)^{h}$. Hence

$$
n=2^{7} \cdot 5^{b} \cdot 17^{c} \cdot 7^{2} \cdot s^{e} \cdot(18913)^{f} \cdot(184417)^{g} \cdot(25646167)^{h}
$$

so that 


$$
3=\frac{\sigma^{* *}(n)}{n}<\frac{255}{128} \cdot \frac{5}{4} \cdot \frac{17}{16} \cdot \frac{50}{49} \cdot \frac{11}{10} \cdot \frac{18913}{18912} \cdot \frac{184417}{184416} \cdot \frac{25646167}{25646166}=2.970031854<3,
$$

a contradiction. Hence $c=14$ is not admissible.

(11) We have

$$
\begin{aligned}
& \sigma^{* *}\left(17^{18}\right)=\{\{2,1\},\{5,2\},\{19,1\},\{29,1\},\{307,1\},\{21881,1\},\{63541,1\}, \\
&\{1270657,1\}\} ; \\
& \sigma^{* *}\left(17^{22}\right)=\{\{2,1\},\{73,1\},\{1321,1\},\{41761,1\},\{72337,1\},\{2141993519227,1\}\} ; \\
& \sigma^{* *}\left(17^{26}\right)=\{\{2,1\},\{5,1\},\{29,1\},\{212057,1\},\{5766433,1\},\{100688449,1\}, \\
& \sigma^{* *}\left(17^{30}\right)=\{\{2,1\},\{257,1\},\{307,1\},\{88741,1\},\{1801601,1\},\{6566760001,1\}, \\
&\{52548582913,1\}\} ; \\
& \sigma^{* *}\left(17^{34}\right)=\{\{2,1\},\{5,1\},\{29,1\},\{37,1\},\{109,1\},\{181,1\},\{2089,1\},\{10949,1\}, \\
&\{83233,1\},\{382069,1\},\{1749233,1\},\{2699538733,1\}\} ; \\
&\{291973723,1\},\{1186844128302568601,1\}\} ; \\
& \sigma^{* *}\left(17^{38}\right)=\{\{2,1\},\{41,1\},\{229,1\},\{1103,1\},\{41761,1\},\{202607147,1\}, \\
&\{25646167,1\},\{256152733,1\},\{940143709,1\},\{6901823633,1\}\} ; \\
&\{26552618219228090162977481,1\}\} ; \\
& \sigma^{* *}\left(17^{42}\right)=\{\{2,1\},\{5,1\},\{29,1\},\{43,1\},\{89,1\},\{307,1\},\{13567,1\},\{25741,1\}, \\
&\{26278001,1\},\{1224199237,1\},\{11330289301,1\},\{13938043025453,1\}\} .
\end{aligned}
$$

From the above it follows from $(3.13 b)$ that for $c=18,22,26,30,34,38,42,46,50, w^{\prime}$ is divisible by at least four odd primes which violates $(3.13 c)$. Hence $c \geq 54$.

Let $s=11$. We note that when $e$ is odd or $4 \mid e$, then $3 \mid \sigma^{* *}\left(11^{e}\right)$. From $(3.13 b)$, it follows that $3 \mid w^{\prime}$. But this not possible. Hence we may assume that $e=2 m$, where $m$ is odd.

(12) If $e=2$. Since $\sigma^{* *}\left(11^{2}\right)=122=2.61$, from (3.13a) and (3.13b), we get

$$
n=2^{7} \cdot 5^{b} \cdot 17^{c} \cdot 7^{2} \cdot 11^{2} \cdot 61^{f} \cdot w^{\prime \prime}
$$

and

$$
2^{5} \cdot 5^{b-3} \cdot 17^{c-1} \cdot 7^{2} \cdot 11^{2} \cdot 61^{f-1} \cdot w^{\prime \prime}=\sigma^{* *}\left(5^{b}\right) \cdot \sigma^{* *}\left(17^{c}\right) \cdot \sigma^{* *}(61)^{f} \cdot \sigma^{* *}\left(w^{\prime \prime}\right),
$$

where

$$
\left(w^{\prime \prime}, 2.3 .5 .7 .11 .17 .61\right)=1 \text { and } w^{\prime \prime} \text { has no more than two odd prime factors. } \quad(3.16 c)
$$


By (b) and (c) of Theorem 3.3, $w^{\prime}$ and consequently $w^{\prime \prime}$ is divisible by primes $p^{\prime}$ and $q^{\prime}$ each exceeding 519. Hence from $(3.16 c), w^{\prime \prime}=\left(p^{\prime}\right)^{g} \cdot\left(q^{\prime}\right)^{h}$, so that

$$
n=2^{7} \cdot 5^{b} \cdot 17^{c} \cdot 7^{2} \cdot 11^{2} \cdot 61^{f} \cdot\left(p^{\prime}\right)^{g} \cdot\left(q^{\prime}\right)^{h} .
$$

We can assume $p^{\prime} \geq 521$ and $q^{\prime} \geq 523$. We have

$$
3=\frac{\sigma^{* *}(n)}{n}<\frac{255}{128} \cdot \frac{5}{4} \cdot \frac{17}{16} \cdot \frac{50}{49} \cdot \frac{122}{121} \cdot \frac{61}{60} \cdot \frac{521}{520} \cdot \frac{523}{522}=2.778188424<3,
$$

a contradiction. Thus, $e=2$ is not admissible.

(13) Let $e=6$. We have $\sigma^{* *}\left(11^{6}\right)=2.7 .19 .7321$. Taking $e=6$ in $(3.13 b)$, we see that $w^{\prime}$ is divisible by 19 and 7321 . We now examine whether $\sigma^{* *}\left(17^{c}\right)$ is divisible by 7321 . Let $p=7321$. Then $r=\operatorname{ord}_{p} 17=2440$. Since $r$ is even and $\ell$ is odd, $p \nmid 17^{\ell}-1$. Also, $r / 2=1220=20.61$. Hence if $p \mid 17^{\ell+1}+1$, then $17^{20}+1 \mid 17^{\ell+1}+1$. We have

$$
17^{20}+1=2.41 .41761 .1186844128302568601 .
$$

It now follows from (3.13b) that $w^{\prime}$ is divisible by the four odd primes $19,7321,41761$ and 1186844128302568601 . This violates $(3.13 c)$. So, $p \nmid 17^{\ell+1}+1$. From (b) and (c) of Theorem 3.3, we infer that 19,7321, $p^{\prime}, q^{\prime}$ are four distinct odd prime factors of $w^{\prime}$. Again this violates $(3.13 c)$. Thus $e=6$ is not admissible.

(14) Let $e=10$. We have $\sigma^{* *}\left(11^{10}\right)=2.5 .13 .61 .1117 .3221$. Taking $e=10$ in (3.13b), we find that $w^{\prime}$ is divisible by four odd primes, namely, 13,61,1117 and 3221. This contradicts $(3.13 c)$. Hence $e=10$ is not admissible.

(15) When $e=14$, we have $\sigma^{* *}\left(11^{e}\right)=2.17 .43 .45319 .6304673$. Since $r=\operatorname{ord}_{43} 17=6$ is even, $43 \nmid 17^{\ell}-1$. Also, $r / 2=3$ is odd. Hence $43 \nmid 17^{\ell+1}+1$.

Let $p=45319$. Then $r^{\prime}=\operatorname{ord}_{p} 17=45318$. Hence $p \nmid 17^{\ell}-1$ since $r^{\prime}$ is even. Also, $r^{\prime} / 2=22654=2.47 .241=94 u^{\prime}$, where $u^{\prime}$ is odd. Hence if $p \mid 17^{\ell+1}+1$, then $17^{94}+1 \mid 17^{\ell+1}+1$. We have

$$
\begin{aligned}
17^{94}+1= & \{\{2,1\},\{5,1\},\{29,1\},\{8837,1\}, \\
& \{179265103693349709880136365395087273880137628400303290349204841 \\
& 92380077903123625556163033417839614972209382061,1\}\} .
\end{aligned}
$$

Taking into account of the factors of $\sigma^{* *}\left(11^{14}\right)$ and $17^{94}+1$, from $(3.13 b)$, we see that $w^{\prime}$ is divisible by six odd prime factors contradicting $(3.13 c)$. Hence $p \nmid 17^{\ell+1}+1$. Let $p^{\prime}$ and $q^{\prime}$ be as given in (b) and (c) of Theorem 3.3. Then from the above discussion either $p^{\prime} \notin\{43,45319,6304673\}$ or $q^{\prime} \notin\{43,45319,6304673\}$. From $(3.13 b)$ it now follows that $w^{\prime}$ is divisible by at least four odd primes contradicting $(3.13 c)$. We conclude that $e=14$ is not admissible. 
(16) We have

$$
\begin{aligned}
\sigma^{* *}\left(11^{18}\right)=\{ & \{2,1\},\{7,1\},\{19,1\},\{61,1\},\{1772893,1\},\{212601841,1\}\} ; \\
\sigma^{* *}\left(11^{22}\right)=\{ & \{2,1\},\{7321,1\},\{10657,1\},\{15797,1\},\{20113,1\},\{1806113,1\}\} ; \\
\left.\sigma^{* *}\left(11^{26}\right)=\{2,1\},\{29,1\},\{61,1\},\{1093,1\},\{1933,1\},\{55527473,1\},\{3158528101,1\}\right\} ; & \\
\sigma^{* *}\left(11^{30}\right)=\{ & \{2,1\},\{5,1\},\{7,1\},\{19,1\},\{3221,1\},\{51329,1\},\{195019441,1\}, \\
\sigma^{* *}\left(11^{34}\right)=\{ & \{2,1\},\{13,1\},\{61,1\},\{1117,1\},\{3138426605161,1\}, \\
& \{50544702849929377,1\}\} ; \\
\sigma^{* *}\left(11^{38}\right)=\{ & \{2,1\},\{41,1\},\{7321,1\},\{1120648576818041,1\}, \\
& \{6115909044841454629,1\}\} ; \\
& \{45319,1\},\{251857,1\},\{2649263870814793,1\}\} ; \\
\sigma^{* *}\left(11^{42}\right)=\{ & \{2,1\},\{7,2\},\{19,1\},\{43,1\},\{61,1\},\{1723,1\},\{8527,1\},\{27763,1\}, \\
& \{28878847,1\},\{1106131489,1\},\{3740221981231,1\}\} ; \\
\sigma^{* *}\left(11^{46}\right)=\{ & \{2,1\},\{17,1\},\{97,1\},\{241,1\},\{829,1\},\{1777,1\},\{6304673,1\}, \\
& \{1856458657451,1\},\{9768997162071483134919121,1\}\} .
\end{aligned}
$$

It now follows that none of $e=18,22,26,30,34,38,42,46$ and 50 is admissible as in each case $w^{\prime}$ will be divisible by at least four odd prime factors (which follows from (3.13b)) contradicting (3.13c).

Thus $e \geq 54$.

We may note that by (b) and (c) of Theorem 3.3, $w^{\prime} \geq 521.523$. Thus if $s=11$,

$$
n=2^{7} \cdot 5^{b} \cdot 17^{c} .7^{2} \cdot 11^{e} \cdot w^{\prime} \geq 128 \cdot 5^{54} \cdot 17^{54} \cdot 11^{54} \cdot 521.523>4.5349 \times 10^{169},
$$

and if $s=13$,

$$
n=2^{7} \cdot 5^{b} \cdot 17^{c} \cdot 7^{2} \cdot 13^{e} \cdot w^{\prime} \geq 128 \cdot 5^{54} \cdot 17^{54} \cdot 13.521 .523>3.43 \times 10^{114} .
$$

This proves (b) of Theorem 3.4. The proof of Theorem 3.4 is complete.

\section{References}

[1] Hagis, P., Jr. (1987). Bi-unitary amicable and multiperfect numbers. The Fibonacci Quarterly, 25(2), 144-150.

[2] Haukkanen, P., \& Sitaramaiah, V. (2020). Bi-unitary multiperfect numbers, I. Notes on Number Theory and Discrete Mathematics, 26(1), 93-171. 
[3] Haukkanen, P., \& Sitaramaiah, V. (2020). Bi-unitary multiperfect numbers, II. Notes on Number Theory and Discrete Mathematics, 26(2), 1-26.

[4] Haukkanen, P., \& Sitaramaiah, V. (2020). Bi-unitary multiperfect numbers, III. Notes on Number Theory and Discrete Mathematics, 26(3), 33-67.

[5] Haukkanen, P., \& Sitaramaiah, V. (2020). Bi-unitary multiperfect numbers, IV(a). Notes on Number Theory and Discrete Mathematics, 26(4), 2-32.

[6] Sándor, J., \& Crstici, P. (2004). Handbook of Number Theory II, Kluwer Academic.

[7] Suryanarayana, D. (1972). The number of bi-unitary divisors of an integer. The Theory of Arithmetic Functions, Lecture Notes in Mathematics 251: 273-282, New York, Springer-Verlag.

[8] Wall, C. R. (1972). Bi-unitary perfect numbers. Proceedings of the American Mathematical Society, 33(1), 39-42. 